\title{
Homogeneous quasi-translations in dimension 5
}

\author{
Michiel de Bondt ${ }^{1}$
}

Received: 8 March 2016 / Accepted: 11 September 2017 / Published online: 13 October 2017 C The Author(s) 2017. This article is an open access publication

\begin{abstract}
We give a proof in modern language of the following result by Paul Gordan and Max Nöther: a homogeneous quasi-translation in dimension 5 without linear invariants would be linearly conjugate to another such quasi-translation $x+H$, for which $H_{5}$ is algebraically independent over $\mathbb{C}$ of $H_{1}, H_{2}, H_{3}, H_{4}$. Just like Gordan and Nöther, we apply this result to classify all homogeneous polynomials $h$ in 5 indeterminates, for which the Hessian determinant is zero. Others claim to have reproved 'the result of Gordan and Nöther in $\mathbb{P}^{4}$, as well, but their proofs have gaps, which can be fixed by using the above result about homogeneous quasi-translations. Furthermore, some of the proofs assume that $h$ is irreducible, which Gordan and Nöther did not. We derive some other properties which $H$ would have. One of them is that $\operatorname{deg} H \geq 15$, for which we give a proof which is less computational than another proof of it by Dayan Liu. Furthermore, we show that the Zariski closure of the image of $H$ would be an irreducible component of $V(H)$, and prove that every other irreducible component of $V(H)$ would be a 3 -dimensional linear subspace of $\mathbb{C}^{5}$ which contains the fifth standard basis unit vector.
\end{abstract}

Keywords Quasi-translation · Hessian · Determinant zero · Homogeneous · Locally nilpotent derivation · Algebraic dependence $\cdot$ Linear dependence

Many of the results of this article appeared already in Chapter 3 in the author's Ph.D. thesis (de Bondt 2009).

M. de Bondt's Ph.D. project was supported by The Netherlands Organization for Scientific Research (NWO).

$凶 \quad$ Michiel de Bondt

M.deBondt@math.ru.nl

1 Department of Mathematics, Radboud University, Postbus 9010, 6500 GL Nijmegen, The Netherlands 
Mathematics Subject Classification 14R05 $\cdot 14 \mathrm{R} 10 \cdot 14 \mathrm{R} 20$

\section{Introduction}

Throughout this paper, we will write $x$ for an $n$-tuple $\left(x_{1}, x_{2}, \ldots, x_{n}\right)$ of variables, where $n$ is a positive integer. We write $\mathcal{J} F$ for the Jacobian matrix of a polynomial map $F=\left(F_{1}, F_{2}, \ldots, F_{m}\right)$ with respect to $x$, where $m$ is another positive integer, i.e.

$$
\mathcal{J} F=\left(\begin{array}{cccc}
\frac{\partial}{\partial x_{1}} F_{1} & \frac{\partial}{\partial x_{2}} F_{1} & \cdots & \frac{\partial}{\partial x_{n}} F_{1} \\
\frac{\partial}{\partial x_{1}} F_{2} & \frac{\partial}{\partial x_{2}} F_{2} & \cdots & \frac{\partial}{\partial x_{n}} F_{2} \\
\vdots & \vdots & & \vdots \\
\frac{\partial}{\partial x_{1}} F_{m} & \frac{\partial}{\partial x_{2}} F_{m} & \cdots & \frac{\partial}{\partial x_{n}} F_{m}
\end{array}\right)
$$

We write $\mathcal{H} f$ for the Hessian matrix of a polynomial $f$ with respect to $x$, i.e.

$$
\mathcal{H} f=\left(\begin{array}{cccc}
\frac{\partial^{2}}{\partial x_{1}^{2}} f & \frac{\partial}{\partial x_{2}} \frac{\partial}{\partial x_{1}} f & \cdots & \frac{\partial}{\partial x_{n}} \frac{\partial}{\partial x_{1}} f \\
\frac{\partial}{\partial x_{1}} \frac{\partial}{\partial x_{2}} f & \frac{\partial^{2}}{\partial x_{2}^{2}} f & \cdots & \frac{\partial}{\partial x_{n}} \frac{\partial}{\partial x_{2}} f \\
\vdots & \vdots & \ddots & \vdots \\
\frac{\partial}{\partial x_{1}} \frac{\partial}{\partial x_{n}} f & \frac{\partial}{\partial x_{2}} \frac{\partial}{\partial x_{n}} f & \cdots & \frac{\partial^{2}}{\partial x_{n}^{2}} f
\end{array}\right)
$$

We see a polynomial $f$ as a polynomial with only one component, so

$$
\mathcal{J} f=\left(\frac{\partial}{\partial x_{1}} f \frac{\partial}{\partial x_{2}} f \cdots \frac{\partial}{\partial x_{n}}\right)
$$

and write $\nabla f=(\mathcal{J} f)^{\mathrm{t}}$. Here, and in the rest of the article, $(\cdots)^{\mathrm{t}}$ stands for the transpose matrix. So

$$
\mathcal{H} f=\mathcal{J}(\nabla f)
$$

Just like with $x$, we will write $y$ for another $n$-tuple $\left(y_{1}, y_{2}, \ldots, y_{n}\right)$ of variables. But unlike $x$ and $y, t$ will be just a single variable.

Definition 1.1 Let $F=x+H$ be a polynomial map from $\mathbb{C}^{n}$ to $\mathbb{C}^{n}$. Then we call $F$ a quasi-translation if $2 x-F=x-H$ is the inverse of $F=x+H$.

The condition that $x-H$ is the inverse of $x+H$ is automatically fulfilled if $\operatorname{deg} H=0$, in which case $x+H$ is a regular translation. So a quasi-translation is a polynomial map which is characterized by a property of a regular translation.

Below are some examples of quasi-translations in dimension $n=4$ : 


$$
\begin{aligned}
& x+\left(x_{2}^{2} x_{3}-3 x_{3}^{3} x_{4}-5,0,0,0\right) \\
& x+\left(1, x_{4}, x_{4}^{2}, 0\right) \\
& x+\left(x_{3}^{2}-3 x_{3}^{3} x_{4}-5, x_{3}+7 x_{4}^{7}, 0,0\right) \\
& x+\left(b\left(a x_{1}-b x_{2}\right), a\left(a x_{1}-b x_{2}\right),\right. \\
& \left.\quad b\left(a x_{3}-b x_{4}\right), a\left(a x_{3}-b x_{4}\right)\right) \quad \text { with } a, b \in \mathbb{C}
\end{aligned}
$$

In the next section, we will show that $x+H$ is a quasi-translation, if and only if $\mathcal{J} H \cdot H=0$. This is equivalent to that for the derivation $D=H_{1} \frac{\partial}{\partial x_{1}}$ $+H_{2} \frac{\partial}{\partial x_{2}}+\cdots+H_{n} \frac{\partial}{\partial x_{n}}, D^{2} x_{i}=0$ for all $i$, because $D^{2} x_{i}=D H_{i}=\mathcal{J} H_{i} \cdot H$.

Hence quasi-translations correspond to a special kind of locally nilpotent derivations. Furthermore, invariants of the quasi-translation $x+H$ are just kernel elements of $D$. Paul Gordan and Max Nöther call these kernel elements 'Functionen $\Phi$ ' in Gordan and Nöther (1876).

In addition, we can write $\exp (D)$ and $\exp (t D)$ for the automorphisms corresponding to the maps $x+H$ and $x+t H$ respectively. But in order to make the article more readable for readers that are not familiar with derivations, we will omit the terminology of derivations further in this article.

Gordan and Nöther (1876) studied (homogeneous) quasi-translations to obtain results about (homogeneous) polynomials $h$ with det $\mathcal{H} h=0$. One such a result is the classification of homogeneous polynomials in 5 indeterminates for which the Hessian determinant is zero. This classification has been reproved in Franchetta (1954) and Garbagnati and Repetto (2009), but only for the case where $h$ is an irreducible polynomial. In Russo (2016, Ch. 7), the proof of Garbagnati and Repetto (2009) is extended to the case where $h$ is a square-free polynomial. With an easy argument, which the reader may find, one can extend these results to the case where $h$ is a power of such a polynomial. But then, you still do not have all polynomials $h$.

However, Russo (2016), told me that by way of de Bondt (2008, Th. 2.2) one can reduce the general case to the case where $h$ is square-free. This is indeed true, because of the following.

Proposition 1.2 Let $h \in \mathbb{C}[x]$ and let $\tilde{h}$ be the square-free part of $h$.

(i) If $\operatorname{det} \mathcal{H} h=0$, then $\operatorname{det} \mathcal{H} \tilde{h}=0$.

(ii) Suppose that $a_{1}, a_{2}, \ldots, a_{n-2} \in \mathbb{C}\left[x_{1}, x_{2}\right]$ are relatively prime. Let

$$
A:=\mathbb{C}\left[x_{1}, x_{2}, a_{1}\left(x_{1}, x_{2}\right) x_{3}+a_{2}\left(x_{1}, x_{2}\right) x_{4}+\cdots+a_{n-2}\left(x_{1}, x_{2}\right) x_{n}\right]
$$

If $\tilde{h} \in A$, then $h \in A$.

\section{Proof}

(i) This is a special case of de Bondt (2008, Th. 2.2).

(ii) Suppose that $\tilde{h} \in A$, and let $f$ be an arbitrary factor of $h$ over $\mathbb{C}[x]$. It suffices to show that $f \in A$.

Over $\mathbb{C}\left(x_{1}, x_{2}\right), h$ is a polynomial in the linear form $a_{1}\left(x_{1}, x_{2}\right) x_{3}+a_{2}\left(x_{1}, x_{2}\right)$. $x_{4}+\cdots+a_{n-2}\left(x_{1}, x_{2}\right) x_{n}$. Just like $\mathbb{C}\left(x_{1}, x_{2}\right)\left[x_{3}\right]$, 


$$
\mathbb{C}\left(x_{1}, x_{2}\right)\left[a_{1}\left(x_{1}, x_{2}\right) x_{3}+a_{2}\left(x_{1}, x_{2}\right) x_{4}+\cdots+a_{n-2}\left(x_{1}, x_{2}\right) x_{n}\right]
$$

is factorially closed in $\mathbb{C}\left(x_{1}, x_{2}\right)\left[x_{3}, x_{4}, \ldots, x_{n}\right]$. Consequently, $f$ is a polynomial over $\mathbb{C}\left(x_{1}, x_{2}\right)$ in the linear form $a_{1}\left(x_{1}, x_{2}\right) x_{3}+a_{2}\left(x_{1}, x_{2}\right) x_{4}+\cdots+$ $a_{n-2}\left(x_{1}, x_{2}\right) x_{n}$ as well.

Take $d \geq 0$ arbitrary, and let $\tilde{f}$ be the part of $f$, which has degree $d$ with respect to $x_{3}, x_{4}, \ldots, x_{n}$. Then $\tilde{f} \in \mathbb{C}[x]$, and over $\mathbb{C}\left(x_{1}, x_{2}\right), \tilde{f}$ is a monomial in the linear form $a_{1}\left(x_{1}, x_{2}\right) x_{3}+a_{2}\left(x_{1}, x_{2}\right) x_{4}+\cdots+a_{n-2}\left(x_{1}, x_{2}\right) x_{n}$. From Gauss's Lemma, it follows that $\tilde{f} \in A$, As $d$ was arbitrary, we can conclude that $f \in A$.

The connection between quasi-translations and polynomial Hessians with determinant zero, which comes from Gordan and Nöther (1876), is given at the beginning of Sect. 4. This connection is used in Garbagnati and Repetto (2009) and Russo (2016, Ch. 7) as well, and appears as Garbagnati and Repetto (2009, p. 33) and Russo (2016, Lem. 7.3.7) respectively. Garbagnati and Repetto (2009) and Russo (2016, Ch. 7) contain classifications in dimensions less than 5 as well, but with the same limitations as above on the factorization of $h$. These limitations are not present in Lossen (2004), which follows the approach of Gordan and Nöther (1876) in proving the classifications in dimensions less than 5.

In Watanabe (2014), it is claimed that rk $\mathcal{J} H \neq 3$ if $x+H$ is a quasi-translation in dimension $n=5$, but this is not true. Hence the proof in Watanabe (2014) of the classification of homogeneous polynomials in 5 indeterminates, for which the Hessian determinant is zero, has a gap. The paper (Franchetta 1954) has an error and hence a gap on the same point. This gap can be fixed by proving that $\mathrm{rk} \mathcal{J} H \neq 3$ indeed, if $x+H$ is associated to a polynomial for which the Hessian determinant is zero, which can be done by way of the results on linear invariants of quasi-translations, as given in Gordan and Nöther (1876) and this paper: see Remark 4.7 at the end of Sect. 4.

Garbagnati and Repetto (2009) and Russo (2016, Ch. 7) on one hand, and de Bondt (2009, Th. 5.3.7) on the other hand, treat the case where rk $\mathcal{J} H=3$ incorrectly as well. But both incorrect treatments are only on subcases which do not overlap, so Russo (2016, Ch. 7) and de Bondt (2009, Th. 5.3.7) fix each other's errors. The error in Garbagnati and Repetto (2009) and Russo (2016, Ch. 7) can be repaired by way of Theorem 4.6, which comes from Gordan and Nöther (1876). The error in de Bondt (2009, Th. 5.3.7) can be repaired by way of Lemma 4.4, which gives a simpler argument than that in Gordan and Nöther (1876).

It is easy to show that for any homogeneous polynomial map $H$ such that rk $\mathcal{J} H=1, x+H$ has $n-1$ independent linear invariants. Gordan and Nöther (1876) proved that any homogeneous quasi-translation $x+H$ such that $\operatorname{rk} \mathcal{J} H=2$ has at least 2 independent linear invariants. In their study of homogeneous quasitranslations $x+H$ in dimension $n=5$ with rk $\mathcal{J} H=3$, Gordan and Nöther (1876) distinguished two cases, namely "Fall a)" and "Fall b)", of which "Fall a)" had two subcases, which we indicate by (a1) and (a2).

The quasi-translations of subcase (a1) in Gordan and Nöther (1876) are the homogeneous quasi-translations $x+H$ in dimension 5 with Jacobian rank three, for which the Zariski closure of the image of $H$ is a 3-dimensional linear subspace of $\mathbb{C}^{5}$. The quasi-translations of case (b) in Gordan and Nöther (1876) are the homogeneous quasi- 
translations in dimension 5 with Jacobian rank three, which are linearly conjugate to another such quasi-translation $x+H$, for which $H_{5}$ is algebraically independent over $\mathbb{C}$ of $H_{1}, H_{2}, H_{3}, H_{4}$, but for which the Zariski closure of the image of $H$ is not a 3-dimensional linear subspace of $\mathbb{C}^{5}$.

The quasi-translations of subcase (a2) in Gordan and Nöther (1876) are categorized by a somewhat technical property, which is the existence of $p^{(1)}$ and $p^{(2)}$ as in (iii) of Theorem 3.8. Let us just say for now that they are the homogeneous quasi-translations in dimension 5 with Jacobian rank three, which do not belong to case (b) or subcase (a1) in Gordan and Nöther (1876). As a consequence of Theorem 3.8, we deduce in Corollary 3.10 that quasi-translations of case (a2) in Gordan and Nöther (1876) have at least one linear invariant, by showing that the linear span of the image of $H$ is 4-dimensional. Having reasoned about these three cases, one can wonder whether they actually exist.

Example 1.3 The following three $H$ 's are chosen in such a way, that $x+H$ with $n=5$ is a quasi-translation which belongs to the above-described case (a1), (a2), and (b), respectively.

(a1) $H=\left(x_{4}^{2}, x_{4} x_{5}, x_{1} x_{5}-x_{2} x_{4}, 0,0\right)$,

(a2) $H=\left(x_{5}^{2}\left(a x_{1}-x_{5}^{2} x_{2}\right), a\left(a x_{1}-x_{5}^{2} x_{2}\right), x_{5}^{2}\left(a x_{3}-x_{5}^{2} x_{4}\right), a\left(a x_{3}-x_{5}^{2} x_{4}\right), 0\right)$ with $a=x_{1} x_{4}-x_{2} x_{3}$,

(b) $H=\left(x_{5}^{5}, b x_{5}^{3}, b^{2} x_{5},-b^{2} x_{1}+2 b x_{2} x_{5}^{2}-x_{3} x_{5}^{4}\right.$, 0) with $b=x_{1} x_{3}-x_{2}^{2}+x_{4} x_{5}$.

The quasi-translations for (a1) and (a2) were found by using techniques of de Bondt (2006, §2). The quasi-translations for (b) was found by applying Propositions 2.4 and 2.5 , on the quasi-translation $x+H$ with $n=4$ and $H=\left(1, x_{4}, x_{4}^{2}, 0\right)$.

An unsolved question is whether a homogeneous quasi-translation in dimension 5 always has a linear invariant or not. We reprove the following results obtained in Gordan and Nöther (1876) in modern language: a homogeneous quasi-translation in dimension 5 without a linear invariant can only belong to case (b) in Gordan and Nöther (1876). Furthermore, we give a somewhat less computational proof of the result in Liu (2011) that a homogeneous quasi-translation in dimension 5 without a linear invariant must have degree 15 at least.

In dimension 6 and up, homogeneous quasi-translations do not need to have linear invariants, see (de Bondt 2006 Th. 2.1). If we substitute $x_{5}=1$ in the quasi-translations of cases (a2) and (b) in Example 1.3 and remove the last component, we get nonhomogeneous quasi-translations in dimension 4 without linear invariants.

The rest of the paper is organized as follows. In the next section, we show some basic concepts about quasi-translations.

In Sect. 3, we prove some geometric results about homogeneous quasi-translations $x+H$ for which rk $\mathcal{J} H \leq(n+1) / 2$. As a consequence, we deduce that a homogeneous quasi-translation in dimension 5 without linear invariants can only belong to case (b) in Gordan and Nöther (1876).

In Sect. 4, we apply the result that a homogeneous quasi-translation in dimension 5 without a linear invariant can only belong to case (b) in Gordan and Nöther (1876), to classify all homogeneous polynomials in 5 indeterminates for which the Hessian determinant vanishes. 
In Sect. 5, we study homogeneous quasi-translations in dimension 5 that belong to case (b) in Gordan and Nöther (1876), with the purpose of getting properties of possible homogeneous quasi-translations in dimension 5 without linear invariants. One of these properties is that the degree of such a quasi-translation is at least 15.

In Sect. 6, we prove some geometric results about quasi-translations which gives us the following result about quasi-translations which belong to case (b) in Gordan and Nöther (1876): the Zariski closure of the image of $H$ is an irreducible component of $V(H)$, which contains a linear 1-dimensional subspace $L$ of $\mathbb{C}^{5}$, such that every other irreducible component of $V(H)$ is a 3-dimensional linear subspace of $\mathbb{C}^{5}$ which contains $L$. Here, $V(H)$ is the set of common zeroes of $H_{1}, H_{2}, \ldots, H_{n}$.

\section{Some basics about quasi-translations}

In Proposition 2.2 below, we will show that quasi-translations are also characterized by $H(x+t H)=H$ and by that $\mathcal{J} H \cdot H$ is the zero vector. We need the following lemma to prove Proposition 2.2.

Lemma 2.1 Assume that $x+H$ is a polynomial map and $f \in \mathbb{C}[x]$. Then

$$
f(x+t H)=f(x)
$$

in case one of the following assumptions is satisfied.

(1) $x+H$ is a quasi-translation and $f(x+H)=f(x)$,

(2) $\mathcal{J} H \cdot H=\left(0^{1}, 0^{2}, \ldots, 0^{n}\right)$ and $\mathcal{J} f \cdot H=0$.

\section{Proof}

(1) Since $(x-H) \circ(x+H)=x$, we see that

$$
\begin{aligned}
(x+m H) \circ(x+H) & =((m+1) x-m(x-H)) \circ(x+H) \\
& =(m+1)(x+H)-m x=x+(m+1) H
\end{aligned}
$$

By induction on $m, x+m H$ is equal to the composition of $m$ copies of $x+H$ for all $m \in \mathbb{N}$. Using $f(x+H)=f(x) m$ times, we obtain

$$
f(x+m H)=f\left((x+H)^{\circ m}\right)=f\left((x+H)^{\circ(m-1)}\right)=\cdots=f(x)
$$

for all $m \in \mathbb{N}$. This is only possible if (2.1) holds.

(2) By the chain rule and $\mathcal{J} H \cdot H=\left(0^{1}, 0^{2}, \ldots, 0^{n}\right)$, we get

$$
\begin{aligned}
\mathcal{J} f(x+t H) \cdot H & =\left.(\mathcal{J} f)\right|_{x=x+t H} \cdot\left(I_{n}+t \mathcal{J} H\right) \cdot H \\
& =\left.(\mathcal{J} f)\right|_{x=x+t H} \cdot H=\frac{\partial}{\partial t} f(x+t H)
\end{aligned}
$$


where $I_{n}$ is the unit matrix of size $n$. Since $\mathcal{J} f \cdot H=0$, it follows from the above that

$$
\mathcal{J}(f(x+t H)-f(x)) \cdot H=\frac{\partial}{\partial t} f(x+t H)
$$

Suppose that $t$ divides the right hand side of (2.2) exactly $r<\infty$ times. Then $t$ divides $f(x+t H)-f(x)$ more than $r$ times. Hence $t$ divides the left hand side of (2.2) more than $r$ times as well, which is a contradiction. So both sides of (2.2) are zero. Since the right hand side of (2.2) is zero, we get (2.1).

Proposition 2.2 Let $H: \mathbb{C}^{n} \rightarrow \mathbb{C}^{n}$ be a polynomial map. Then the following properties are equivalent:

(1) $x+H$ is a quasi-translation,

(2) $H(x+t H)=H$ (where $t$ is a variable $)$,

(3) $\mathcal{J} H \cdot H=\left(0^{1}, 0^{2}, \ldots, 0^{n}\right)$.

Furthermore, if any of (1), (2) and (3) is satisfied, then

$$
f(x+H)=f(x) \Longleftrightarrow f(x+t H)=f(x) \Longleftrightarrow \mathcal{J} f \cdot H=0
$$

for all $f \in \mathbb{C}[x]$, and

$$
\left.(\mathcal{J} H)\right|_{x=x-t \mathcal{J} H}=(\mathcal{J} H)+t(\mathcal{J} H)^{2}+t^{2}(\mathcal{J} H)^{3}+\cdots
$$

Proof The middle hand side of (2.3) gives the left hand side by substituting $t=1$ and the right hand side by taking the coefficient of $t^{1}$. Lemma 2.1 gives the converse implications by way of (1) and (3). Hence (2.3) follows as soon as we have the equivalence of (1), (2) and (3).

By taking the Jacobian of (2), we get $\left.(\mathcal{J} H)\right|_{x=x+t H} \cdot\left(I_{n}+t \mathcal{J} H\right)=\mathcal{J} H$, which gives (2.4) after substituting $t=-t$. Therefore, it remains to show that (1), (2) and (3) are equivalent.

(1) $\Rightarrow$ (2) Assume (1). Since $x=(x-H) \circ(x+H)=x+H-H(x+H)$, we see that $H(x+H)=H$, and (2) follows by taking $f=H_{i}$ for each $i$ in (1) of Lemma 2.1.

$(2) \Rightarrow(1)$ Assume (2). Then

$$
\begin{aligned}
(x-t H) \circ(x+t H) & =(x+t H)-t H(x+t H) \\
& =x+t H-t H=x
\end{aligned}
$$

which gives (1) after substituting $t=1$.

(2) $\Rightarrow$ (3) Assume (2). By taking the coefficient of $t^{1}$ of (2), we get (3).

(3) $\Rightarrow$ (2) Assume (3). By taking $f=H_{i}$ in (2) of Lemma 2.1, we get (2).

Proposition 2.3 below gives a tool to obtain quasi-translations $x+H$ over $\mathbb{C}$ for which $\operatorname{gcd}\left\{H_{1}, H_{2}, \ldots, H_{n}\right\}=1$ from arbitrary quasi-translations $x+H$ over $\mathbb{C}$. 
Proposition 2.3 Assume $x+g H$ is a quasi-translation over $\mathbb{C}$, where $g \in \mathbb{C}[x]$ is nonzero. Then $x+H$ is a quasi-translation over $\mathbb{C}$ as well. Furthermore, the invariants of $x+H$ and $x+g H$ are the same. If additionally $H$ is homogeneous of positive degree, then $\mathrm{rk} \mathcal{J} g H=\operatorname{rk} \mathcal{J} H$.

Proof By $(1) \Rightarrow(2)$ of Proposition 2.2, we see that $g(x+\operatorname{tg} H) \cdot H_{i}(x+\operatorname{tg} H)=g \cdot H_{i}$. We can substitute $t=g^{-1} t$ in it, to obtain that

$$
\begin{aligned}
\operatorname{deg}_{t} H_{i}(x+t H) & \leq \operatorname{deg}_{t} g(x+t H)+\operatorname{deg}_{t} H_{i}(x+t H) \\
& =\operatorname{deg}_{t}\left(g H_{i}\right)(x+t H) \leq 0
\end{aligned}
$$

for each $i$, which is exactly $H(x+t H)=H$. Hence $x+H$ is a quasi-translation on account of $(2) \Rightarrow$ (1) of Proposition 2.2.

Assume $f$ is an invariant of $x+H$. Then $f(x+t H)=f(x)$ on account of (2.3), and by substituting $t=g$ we see that $f$ is an invariant of $x+g H$. The converse follows in a similar manner by substituting $t=g^{-1}$.

Suppose that $H$ is homogeneous of positive degree. From Proposition 1.2.9 of either van den Essen (2000) or de Bondt (2009), we deduce that in order to prove that rk $\mathcal{J} g H=$ rk $\mathcal{J} H$, it suffices to show that $\operatorname{trdeg}_{\mathbb{C}} \mathbb{C}(g H)=\operatorname{trdeg}_{\mathbb{C}} \mathbb{C}(H)$. For that purpose, we show that for any $R \in \mathbb{C}[y]$, both $R(g H)$ and $R(H)$ are zero if one of them is.

Suppose that either $R(g H)=0$ or $R(H)=0$ for some $R \in \mathbb{C}[y]$, say of degree $r$. Let $\bar{R}$ be the leading homogeneous part of $R$. If $R(H)=0$, then $\bar{R}(H)=0$ because $H$ is homogeneous of positive degree. If $R(g H)=0$, then $\operatorname{deg} \bar{R}(g H)<$ $r \operatorname{deg} g H=\operatorname{deg} g^{r}+r \operatorname{deg} H$, so $\operatorname{deg} \bar{R}(H)<r \operatorname{deg} H$, which is only possible if $\bar{R}(H)=0$. So $\bar{R}(g H)=\bar{R}(H)=0$ in any case. Hence either $(R-\bar{R})(g H)=0$ or $(R-\bar{R})(g H)=0$. By induction to the number of homogeneous parts of $R$, it follows that $R(g H)=R(H)=0$ indeed.

Proposition 2.4 gives a criterion about preservation of the quasi-translation property with respect to conjugation with an invertible polynomial map.

Proposition 2.4 Assume $x+H$ is a quasi-translation in dimension $n$ over $\mathbb{C}$, and $F$ is an invertible polynomial map in dimension $n$ over $\mathbb{C}$ with inverse $G$. Then

$$
G \circ(x+H) \circ F
$$

is a quasi-translation as well, if and only if $\operatorname{deg}_{t} G_{i}(x+t H) \leq 1$ for all $i$. In particular, if $T$ is an invertible matrix of size $n$ over $\mathbb{C}$, we have that

$$
x+T^{-1} H(T x)=T^{-1}(T x+H(T x))=T^{-1} x \circ(x+H) \circ T x
$$

is a quasi-translation as well.

Proof Assume first that $\operatorname{deg}_{t} G_{i}(x+t H) \leq 1$ for all $i$. Then we can write

$$
G(x+t H)=G^{(0)}+t G^{(1)}
$$


Notice that $G^{(0)}=\left.G(x+t H)\right|_{t=0}=G$. Hence

$$
G \circ(x+t H) \circ F=G^{(0)}(F)+t G^{(1)}(F)=G(F)+t G^{(1)}(F)=x+t G^{(1)}(F)
$$

By substituting $t=1$ on both sides, we obtain that $G \circ(x+H) \circ F=x+G^{(1)}(F)$ and substituting $t=-1$ tells us that its inverse $G \circ(x-H) \circ F$ is equal to $x-G^{(1)}(F)$. Thus $G \circ(x+H) \circ F$ is a quasi-translation indeed.

Assume next that $G \circ(x+H) \circ F$ is a quasi-translation $x+\tilde{H}$. Then $x-\tilde{H}$ is the inverse of $G \circ(x+H) \circ F$, which is $G \circ(x-H) \circ F$. Hence

$$
\tilde{H}=(G \circ(x+H) \circ F)-x=x-(G \circ(x-H) \circ F)
$$

Substituting $x=G(x+m H)$ in the above gives

$$
\begin{aligned}
& G(x+m H+H(x+m H))-G(x+m H)=G(x+m H) \\
& -G(x+m H-H(x+m H))
\end{aligned}
$$

Since $H(x+m H)=H$ on account of $(1) \Rightarrow(2)$ of Proposition 2.2, we obtain

$$
G(x+(m+1) H)-G(x+m H)=G(x+m H)-G(x+(m-1) H)
$$

By induction on $m$, we get $G(x+(m+1) H)-G(x+m H)=G(x+H)-G(x)$ for all $m \in \mathbb{N}$, whence

$$
\begin{aligned}
G(x+\tilde{m} H)-G(x) & =\sum_{m=0}^{\tilde{m}-1} G(x+(m+1) H)-G(x+m H) \\
& =\tilde{m}(G(x+H)-G(x))
\end{aligned}
$$

for all $\tilde{m} \in \mathbb{N}$. This is only possible if $G(x+t H)-G(x)=t(G(x+H)-G(x))$. Hence $\operatorname{deg}_{t} G(x+t H) \leq 1$, as desired.

Proposition 2.5 gives a tool to obtain homogeneous quasi-translations over $\mathbb{C}$ from arbitrary quasi-translations $x+H$ over $\mathbb{C}$. Hence we can obtain results about arbitrary quasi-translations by studying homogeneous ones.

Proposition 2.5 Assume $x+H$ is a quasi-translation over $\mathbb{C}$ in dimension $n$, and

$$
d \geq \operatorname{deg} H:=\max \left\{\operatorname{deg} H_{1}, \operatorname{deg} H_{2}, \ldots, \operatorname{deg} H_{n}\right\}
$$

Then

$$
\left(x, x_{n+1}\right)+x_{n+1}^{d}\left(H\left(x_{n+1}^{-1} x\right), 0\right)
$$

is a homogeneous quasi-translation over $\mathbb{C}$ in dimension $n+1$. 
Proof Denote

$$
\left(x, x_{n+1}\right)=: \tilde{x} \quad \text { and } \quad x_{n+1}^{d}\left(H\left(x_{n+1}^{-1} x\right), 0\right)=: \tilde{H}
$$

We must show that $\tilde{x}+\tilde{H}$ is a quasi-translation in dimension $n+1$ over $\mathbb{C}$. On account of $(3) \Rightarrow(1)$ of Proposition 2.2, it suffices to show that $\mathcal{J}_{\tilde{x}} \tilde{H} \cdot \tilde{H}=\left(0^{1}, 0^{2}, \ldots, 0^{n+1}\right)$. Since $\tilde{H}_{n+1}=0$, this is equivalent to

$$
\mathcal{J} \tilde{H} \cdot x_{n+1}^{d} H\left(x_{n+1}^{-1} x\right)=\left(0^{1}, 0^{2}, \ldots, 0^{n+1}\right)
$$

Using that $\mathcal{J} \tilde{H}_{n+1}$ is the zero row, we see that it suffices to show that

$$
\mathcal{J}\left(x_{n+1}^{d} H\left(x_{n+1}^{-1} x\right)\right) \cdot x_{n+1}^{d} H\left(x_{n+1}^{-1} x\right)=\left(0^{1}, 0^{2}, \ldots, 0^{n}\right)
$$

This is indeed the case, because the chain rule tells us that

$$
\begin{aligned}
\left(0^{1}, 0^{2}, \ldots, 0^{n}\right) & =x_{n+1}^{2 d-1} \cdot\left(0^{1}, 0^{2}, \ldots, 0^{n}\right) \\
& =x_{n+1}^{2 d-1} \cdot(\mathcal{J} H \cdot H)_{x=x_{n+1}^{-1} x} \\
& =x_{n+1}^{2 d-1} \cdot\left(\mathcal{J} H \cdot x_{n+1}^{-1} \cdot x_{n+1} H\right)_{x=x_{n+1}^{-1} x} \\
& =x_{n+1}^{2 d-1} \cdot \mathcal{J}\left(H\left(x_{n+1}^{-1} x\right)\right) \cdot x_{n+1} H\left(x_{n+1}^{-1} x\right) \\
& =\mathcal{J}\left(x_{n+1}^{d} H\left(x_{n+1}^{-1} x\right)\right) \cdot x_{n+1}^{d} H\left(x_{n+1}^{-1} x\right)
\end{aligned}
$$

Proposition 2.6 below connects quasi-translations with homogeneity.

Proposition 2.6 Assume $H$ is a homogeneous polynomial map over $\mathbb{C}$. Then the assertions

(1) $\mathcal{J} H^{2}$ is the zero matrix,

(2) $x+H$ is a quasi-translation,

(3) $H(H)=\left(0^{1}, 0^{2}, \ldots, 0^{n}\right)$ and $\mathrm{rk} \mathcal{J} H \leq \max \{n-2,1\}$,

satisfy $(1) \Rightarrow(2) \Rightarrow(3)$.

Proof Suppose that $H$ is homogeneous of degree $d$. Let $E: \mathbb{C}[x]^{n} \rightarrow \mathbb{C}[x]^{n}$ be the map which multiplies each term in any of the $n$ components by its own degree. Then one can verify that $E(H)=\mathcal{J} H \cdot x$. So $\mathcal{J} H \cdot H=d^{-1} \mathcal{J} H \cdot d H=d^{-1} \mathcal{J} H \cdot E(H)$ $=d^{-1} \mathcal{J} H^{2} \cdot x$. Hence $(1) \Rightarrow$ (2) follows from (3) $\Rightarrow$ (1) of Proposition 2.2.

In order to prove $(2) \Rightarrow(3)$, assume that (2) holds. By looking at the coefficient of $t^{d}$ of $H(x+t H)-H(x)$, we deduce that $H(H)=\left(0^{1}, 0^{2}, \ldots, 0^{n}\right)$, which is the first claim of (3).

To show the second claim of (3), assume that rk $\mathcal{J} H>1$. Write $H=g \tilde{H}$, where $g \in \mathbb{C}[x]$, such that $\operatorname{gcd}\left\{\tilde{H}_{1}, \tilde{H}_{2}, \ldots, \tilde{H}_{n}\right\}=1$. Since rk $\mathcal{J} H>1$, we have $\operatorname{deg} \tilde{H} \geq 1$. Furthermore, $V(\tilde{H})$ cannot be written as a zero set of a single polynomial. Since $\mathbb{C}[x]$ is a unique factorization domain, we see that $\operatorname{dim} V(\tilde{H}) \leq n-2$. 
Using Proposition 2.3, Proposition 1.2.9 of either van den Essen (2000) or de Bondt (2009), and the above obtained $\tilde{H}(\tilde{H})=0$ and $\operatorname{dim} V(\tilde{H}) \leq n-2$, in that order, we deduce that

$$
\operatorname{rk} \mathcal{J} H=\operatorname{rk} \mathcal{J} \tilde{H}=\operatorname{trdeg}_{\mathbb{C}} \mathbb{C}(\tilde{H}) \leq \operatorname{dim} V(\tilde{H}) \leq n-2
$$

which gives the second claim of (3).

\section{The image of the map $H$ of quasi-translations $x+H$}

We prove several results about quasi-translations with geometrical arguments. Some of these results have been claimed by Gordan and Nöther (1876). For the last two sections, we need several parts of Corollary 3.10 in this section.

Since the results may essentially be useful for non-homogeneous quasi-translations as well, it does not seem to be a good idea to work with projective varieties. But we will need the completeness of complex projective space in some manner. The lemma below gives us an affine version of that.

Lemma 3.1 Let $\tilde{Z} \subseteq \mathbb{C}^{m+k n}$ be closed with respect to the Euclidian topology. Assume that for every point of $\tilde{Z}$, the projection onto its last $k n$ coordinates gives a point of $\mathbb{C}^{k n}$ with complex norm $\sqrt{k}$. Let $\tilde{X}$ be the image of the projection of $\tilde{Z}$ onto its first $m$ coordinates.

Suppose that there is an irreducible variety $X \subseteq \mathbb{C}^{m}$ and a Zariksi open set $U$ of $X$, such that $U \subseteq \tilde{X} \subseteq X$. Then $\tilde{X}=X$.

Proof Since the set of points in $\mathbb{C}^{k n}$ whose complex norm is $\sqrt{k}$ form a compact space, the projection of $\tilde{Z}$ onto $\tilde{X}$ is closed with respect to the Euclidean topology. Hence $\tilde{X}$ is closed in the Euclidean topology. So $\tilde{X}$ contains the Euclidean closure of $U$ in $X$. On account of O'Meara et al. (2011, Th. 7.5.1), the Euclidean closure of $U$ in $X$ is the same as the Zariksi closure of $U$ in $X$, which is $X$. Hence $X \subseteq \tilde{X}$. So $\tilde{X}=X$ indeed.

Notice that reverting to Euclidean topology is not only because the complex inner product cannot be expressed as a polynomial, but also because the Zariski topology of a product is not the corresponding product topology.

We also need a weak form of the projective fiber dimension theorem in some manner. Lemma 3.3 below is an affine version of that. But first, we need another lemma.

Lemma 3.2 Suppose that $H \in \mathbb{C}[x]^{n}$. Then the Zariski closure $W$ of the image of $H$ is irreducible and has dimension $\mathrm{rk} \mathcal{J} H$.

Furthermore, $V(H)$ has dimension at least $n-\mathrm{rk} \mathcal{J} H$ if $H$ has no constant part.

Proof From Proposition 1.2.9 of either van den Essen (2000) or de Bondt (2009), it follows that $\mathrm{rk} \mathcal{J} H=\operatorname{trdeg}_{\mathbb{C}} \mathbb{C}(H)$. Hence $\operatorname{dim} W=\operatorname{rk} \mathcal{J} H$ indeed.

Let $Z$ be a component of $W$ and let $Y$ be the union of the other components of $W$. By definition of $Z, U:=H^{-1}(W \backslash Y) \neq \varnothing$. By continuity of $H, U$ is open and $H^{-1}(Z) \supseteq U$ is closed, so $H^{-1}(Z)=\mathbb{C}^{n}$ and $W=Z$ is irreducible. 
To prove the last claim, suppose that $H$ has no constant part. Then $0 \in V(H)$. From a weak version of the affine fiber dimension theorem (or from Lemma 3.3 below, applied on the map $\left.\left(H, x_{n+1}\right)\right)$, it follows that $\operatorname{dim} V(H)=\operatorname{dim} H^{-1}(0) \geq n-\mathrm{rk} \mathcal{J} H$ indeed.

Lemma 3.3 Suppose that $H: \mathbb{C}^{n} \rightarrow \mathbb{C}^{n}$ is a polynomial map and $p \in \mathbb{C}^{n}$, such that the linear span $\mathbb{C} p$ of $p$ contains infinitely many points of the image of $H$. Then there exists an irreducible component $X$ of $H^{-1}(\mathbb{C} p)$ such that $H(X)$ has infinitely many points, and the dimension of any such $X$ is larger than $n-\operatorname{rk} \mathcal{J} H$.

Proof Let $W$ be the Zariski closure of the image of $H$. On account of Lemma 3.2, $\operatorname{dim} W=\operatorname{rk} \mathcal{J} H$. Take a generic linear subspace $L \ni p$ of dimension $n+1-\operatorname{rk} \mathcal{J} H$ of $\mathbb{C}^{n}$, so that $\operatorname{dim}(L \cap W)=1$. The set $Y:=\left\{c \in \mathbb{C}^{n} \mid H(c) \in L\right\}$ is the zero set of rk $\mathcal{J} H-1 \mathbb{C}$-linear forms in the components of $H$. By applying (Hartshorne 1977 Ch. I, Prop. 7.1) rk $\mathcal{J} H-2$ times, it follows that every irreducible component of $Y$ have dimension greater than $n-$ rk $\mathcal{J} H$. Furthermore, $\operatorname{dim} H(Y)=1$ because $H(Y)=L \cap W$.

Since $\mathbb{C} p \cap H(Y)$ contains infinitely many points and $Y$ has finitely many irreducible components, there is an irreducible component $X$ of $Y$ such that $H(X)$ has infinitely many points of $\mathbb{C} p$. Furthermore, $\operatorname{dim} X>n-$ rk $\mathcal{J} H$, because all irreducible components of $Y$ have dimension greater than $n-\mathrm{rk} \mathcal{J} H$. So it remains to show that $X \subseteq H^{-1}(\mathbb{C} p)$.

Since $H(X)$ has infinitely many points of $\mathbb{C} p$, it follows that $\mathbb{C} p$ is contained in the Zariski closure of $H(X)$. As $\operatorname{dim} H(X) \leq \operatorname{dim} H(Y)=1=\operatorname{dim} \mathbb{C} p, \mathbb{C} p$ is a component of the Zariski closure of $H(X)$. Now $X \subseteq H^{-1}(\mathbb{C} p)$ follows in a similar manner as $\mathbb{C}^{n} \subseteq H^{-1}(Z)$ in the proof of Lemma 3.2.

Lemma 3.4 Assume $x+H$ is a homogeneous quasi-translation over $\mathbb{C}$. Suppose that $p$ and $q$ are independent and contained in the image of $H$. Then there exists an algebraic set $X$ of dimension at least $n-2(\operatorname{rk} \mathcal{J} H-1)$, such that $H(c+t p)=H(c+t q)=0$ for all $c \in X$.

Proof On account of Lemma 3.3, there exist irreducible algebraic sets $X_{p}$ and $X_{q}$ of dimension at least $n+1-\mathrm{rk} \mathcal{J} H$, such that $H\left(X_{p}\right)$ and $H\left(X_{q}\right)$ contain infinitely many points of $\mathbb{C} p$ and $\mathbb{C} q$ respectively. The set $X_{p} \cap H^{-1}\left(\mathbb{C}^{*} p\right)$ is an open subset of $X_{p}$, and its Zariski closure is just $X_{p}$ because $X_{p}$ is irreducible. For $c \in H^{-1}\left(\mathbb{C}^{*} p\right)$, we have $H(c+t p)=H(c)=\lambda p$ for some $\lambda \in \mathbb{C}$ on account of (1) $\Rightarrow$ (2) of Proposition 2.2. Hence $H(c+t p)=H(c) \in \mathbb{C} p$ for every $c \in X_{p}$.

By a similar argument with $q$ instead of $p$, we see that $H(c+t p)=H(c)=$ $H(c+t q)$ is dependent of both $p$ and $q$ for every $c \in X_{p} \cap X_{q}$. Due to the homogeneity of $H, 0 \in X_{p} \cap X_{q}$. Hence it follows from Hartshorne (1977, Ch. I, Prop. 7.1) that the dimension of $X_{p} \cap X_{q}$ is at least $n-2($ rk $\mathcal{J} H-1)$. So $X=X_{p} \cap X_{q}$ suffices.

Lemma 3.5 Assume $x+H$ is a homogeneous quasi-translation in dimension $n \leq 5$ over $\mathbb{C}$, such that $\mathrm{rk} \mathcal{J} H=2$ and $\operatorname{dim} V(H) \leq n-2$. Then $V(H)$ contains the linear span of the image of $H$. 
Proof $V(H)$ contains only finitely many $(n-2)$-dimensional linear subspaces of $\mathbb{C}^{n}$ because $\operatorname{dim} V(H) \leq n-2$. Furthermore, the Zariski closure of the image of $H$ is irreducible on account of Lemma 3.2. From those two facts, we can deduce that it suffices to show that every nonzero $p$ in the image of $H$ is contained in an $(n-2)$-dimensional linear subspace of $\mathbb{C}^{n}$ which is contained in $V(H)$.

So take any nonzero $p$ in the image of $H$. Take $q$ independent of $p$ such that $q$ is the image of $H$ as well. From Lemma 3.4, it follows that there exists an algebraic set $X$ of dimension at least $n-2(\mathrm{rk} \mathcal{J} H-1)=n-2$, such that $H(c+t p)=H(c+t q)=0$ for all $c \in X$. Choose $X$ irreducible. Since $\operatorname{dim} V(H) \leq n-2$ and $X \subseteq V(H)$, it follows that $\operatorname{dim} X=n-2$ and that the interior $X^{\circ}$ of $X$ as a closed subset of $V(H)$ is nonempty.

Take $c \in X^{\circ}$, such that $c$ is independent of $p$ and $q$ if $n=5$. Then the linear span of $c, p$ and $q$ has dimension at least $\max \{2, n-2\}$. Since $H(c+t p)=0$, the linear span $L$ of $c$ and $p$ is contained in $V(H)$. Since $c \in L \subseteq V(H)$ and $c \in X^{\circ}$, it follows from the irreducibility of $L$ that $L \subseteq X$.

In as similar manner, it follows that for every $\tilde{c} \in L \cap X^{\circ}$, hence for all $\tilde{c} \in L$, the linear span of $\tilde{c}$ and $q$ is contained in $V(H)$. So the linear span of $L$ and $q$ is contained in $V(H)$. This linear span has dimension at least $\max \{2, n-2\}$. Since $\operatorname{dim} V(H) \leq n-2$, it follows that $n \geq 4$ and that $p$ is contained in an $(n-2)$-dimensional linear subspace of $\mathbb{C}^{n}$ which is contained in $V(H)$.

Theorem 3.6 (Gordan and Nöther) Assume $x+H$ is a homogeneous quasi-translation over $\mathbb{C}$, such that $\operatorname{deg} H \geq 1$.

(i) If $\mathrm{rk} \mathcal{J} H \leq 1$, then the image of $H$ is a line through the origin and $x+H$ has $n-1$ independent linear invariants.

(ii) If $\operatorname{gcd}\left\{H_{1}, H_{2}, \ldots, H_{n}\right\}=1$, then $2 \leq \operatorname{rk} \mathcal{J} H \leq \operatorname{dim} V(H) \leq n-2$.

(iii) If rk $\mathcal{J} H=2$, then $x+H$ has at least two independent linear invariants.

Proof For the moment, we prove (iii) only for the case where $n \leq 5$, because we do not need the case where $n \geq 6$ in this paper. To prove the general case of (iii), one can replace the use of Lemma 3.5 by that of the more general Corollary 6.5 in the last section.

Let $W$ be the Zariski closure of the image of $H$. From Lemma 3.2, it follows that $W$ is irreducible and that $\operatorname{dim} W=\operatorname{rk} \mathcal{J} H$.

(i) As $\operatorname{deg} H \geq 1$, the case rk $\mathcal{J} H=0$ is impossible. So assume that rk $\mathcal{J} H=$ 1. Since $H$ is homogeneous and $\operatorname{dim} W=\operatorname{rk} \mathcal{J} H=1$, it follows from the irreducibility of $W$ that the image of $H$ can only be a line through the origin. Hence there are $n-1$ independent linear forms $l_{1}, l_{2}, \ldots, l_{n-1}$ which vanish on the image of $H$. So $l_{1}, l_{2}, \ldots, l_{n-1}$ are invariants of $x+H$.

(ii) Assume that $\operatorname{gcd}\left\{H_{1}, H_{2}, \ldots, H_{n}\right\}=1$. Since deg $H \geq 1$, it follows from (i) that rk $\mathcal{J} H \geq 2$. From (2) $\Rightarrow$ (3) of Proposition 2.6, it follows that rk $\mathcal{J} H \leq n-2$, but its proof tells us that even $\operatorname{rk} \mathcal{J} H \leq \operatorname{dim} V(H) \leq n-2$. So $2 \leq \operatorname{rk} \mathcal{J} H \leq$ $\operatorname{dim} V(H) \leq n-2$.

(iii) Assume that rk $\mathcal{J} H=2$. From Lemma 3.2, it follows that $\operatorname{dim} V(H) \geq$ $n-\operatorname{rk} \mathcal{J} H=n-2$. Write $H=g \tilde{H}$, where $g \in \mathbb{C}[x]$, such that $\operatorname{gcd}\left\{\tilde{H}_{1}, \tilde{H}_{2}, \ldots, \tilde{H}_{n}\right\}=1$. Since $\operatorname{rk} \mathcal{J} H=2>1$, we have $\operatorname{deg} \tilde{H} \geq 1$. 
On account of Proposition 2.3, rk $\mathcal{J} \tilde{H}=$ rk $\mathcal{J} H=2$. Furthermore, $2 \leq$ $\operatorname{dim} V(\tilde{H}) \leq n-2$ on account of (ii), so $n \geq 4$.

From Lemma 3.5, it follows that the linear span of the image of $\tilde{H}$ is contained in $V(\tilde{H})$. Since $\operatorname{dim} V(\tilde{H}) \leq n-2$, the linear span of the image of $\tilde{H}$ has dimension at most $n-2$ as well. Hence there are at least two independent linear forms $l_{1}$ and $l_{2}$ which vanish on the image of $\tilde{H}$. Thus $l_{i}(\tilde{H})=0$ and $l_{i}(H)=g \cdot 0=0$ for both $i \leq 2$. So $l_{1}$ and $l_{2}$ are invariants of $x+H$.

Definition 3.7 Let $H$ be a polynomial map. We define a $G N$-plane of $H$ as a 2dimensional linear subspace of $\mathbb{C}^{n}$ which is contained in $V(H)$.

Theorem 3.8 Assume $x+H$ is a homogeneous quasi-translation over $\mathbb{C}$, such that $2 \leq \mathrm{rk} \mathcal{J} H \leq(n+1) / 2$. Write $W$ for the Zariski closure of the image of $H$.

(i) For each $p \in W$ and each $q \in W$, there are GN-planes $L_{p} \ni p$ and $L_{q} \ni q$ of $H$ which intersect nontrivially.

(ii) If there exists a $p \in W$ which is contained in only finitely many GN-planes of $H$, then the set of such $p \in W$ is not contained in a proper algebraic subset of $W$.

(iii) Suppose that $p^{(1)}, p^{(2)}, \ldots, p^{(k)} \in W$, such that $p^{(i)}$ is contained in only finitely many $G N$-planes of $H$ for each $i$.

Then there exist $G N$-planes $L_{p^{(1)}} \ni p^{(1)}, L_{p^{(2)}} \ni p^{(2)}, \ldots, L_{p^{(k)}} \ni p^{(k)}$ of $H$, such that for each $q \in W$, there exists a GN-plane $L_{q} \ni q$ of $H$ which intersects $L_{p^{(i)}}$ nontrivially for each $i$.

Proof

(i) We first show that (i) holds for all $(p, q)$ in a dense open subset of $W^{2}$. The generic property of $p$ and $q$ that we assume is that $p$ and $q$ are independent and contained in the image of $H$ itself. From Mumford (1999, §1.8, Th. 3), it follows that the image of $H$ contains an open subset of $W$, so that we can easily show that we are considering a dense open subset of $W^{2}$ indeed. From Lemma 3.4, it follows that there exists an algebraic set $X$ of dimension at least $n-2(\operatorname{rk} \mathcal{J} H-1) \geq 1$, such that $H(c+t p)=H(c+t q)=0$ for every $c \in X$. Take $c \in X$ nonzero. Since $H$ is homogeneous, we deduce by substituting $t=t^{-1}$ that $H(t c+p)=H(t c+q)=0$.

In the general case, consider the sets

$Z:=\left\{(p, q, c, b) \in W^{2} \times\left(\mathbb{C}^{n}\right)^{2} \mid H(t c+p)=H(t c+q)=0\right.$ and $\left.b^{\mathrm{t}} c=1\right\}$

and

$$
\tilde{Z}:=\{(p, q, c, b) \in Z \mid b \text { is the complex conjugate of } c\}
$$

By applying proper substitutions in $t$, we see that the image $\tilde{X}$ of the projection of $\tilde{Z}$ onto its first $2 n$ coordinates is equal to that of $Z$. Since $\tilde{X}$ contains an open subset of $X:=W \times W$, it follows from Lemma 3.1 that $\tilde{X}=X$, which gives (i).

(ii) Suppose that there exists a $p \in W$ for which there are only finitely many GNplanes $L_{p} \ni p$. Let $Y$ be the set of $q \in W$ for which there are infinitely many 
GN-planes $L_{q} \ni q$. It is clear that (ii) holds if $Y=\{0\}$, so assume that there exist a $q \in Y$ which is nonzero. Take $P:=\{c \in V(H) \mid H(c+t p)=0\}$ and $Q:=\{c \in V(H) \mid H(c+t q)=0\}$. Since $H$ is homogeneous, we see that both $P$ and $Q$ are unions of GN-planes. Furthermore, $\operatorname{dim} P=2$ and $\operatorname{dim} Q \geq 3$ because of the cardinality assumptions on the GN-planes in $P$ and $Q$.

Let $L$ be a generic linear subspace of dimension $n-2$ of $\mathbb{C}^{n}$, so that $\operatorname{dim}(L \cap P)=$ 0 . Then $L \cap P=\{0\} \subseteq L \cap Q$ and on account of Hartshorne (1977, Ch. I, Prop. 7.1), $\operatorname{dim}(L \cap Q) \geq 1$. Now define

$$
Z:=\left\{(r, c, b) \in W \times L \times \mathbb{C}^{n} \mid H(t c+r)=0 \text { and } b^{\mathrm{t}} c=1\right\}
$$

and

$$
\tilde{Z}:=\{(r, c, b) \in Z \mid b \text { is the complex conjugate of } c\}
$$

By applying proper substitutions in $t$, we see that the image $\tilde{X}$ of the projection of $\tilde{Z}$ onto its first $n$ coordinates is equal to that of $Z$. Furthermore $q$ is contained in $\tilde{X}$, but $p$ is not. Since $q \in Y \backslash\{0\}$ was arbitrary, we see that $Y \subseteq \tilde{X}$.

If $Y$ would contain an open subset of $W$, then Lemma 3.1 tells us that $\tilde{X}=W$, which contradicts that $p$ is not contained in $\tilde{X}$. So $Y$ does not contain an open subset of $W$, and $W \backslash Y$ is not contained in a proper closed subset of $W$ indeed.

(iii) We can simplify (iii) by changing both the quantization set of $q$ and the quantization order, to get the following.

(iii') Suppose that $p^{(1)}, p^{(2)}, \ldots, p^{(k)} \in W$, such that $p^{(i)}$ is contained in only finitely many GN-planes of $H$ for each $i$.

Then for each $q \in W$ which contains only finitely many GN-planes of $H$, there exist a GN-plane $L_{q}$ of $H$ and GN-planes $L_{p^{(1)}} \ni p^{(1)}, L_{p^{(2)}} \ni p^{(2)}$, $\ldots, L_{p^{(k)}} \ni p^{(k)}$ of $H$, such that $L_{q}$ and $L_{p^{(i)}}$ intersect nontrivially for each $i$.

The case where $k=1$ of this simplification follows from (i). The case where $k \geq 2$ of this simplification follows from the case where $k=1$ of the unsimplified (iii) with $p^{(1)}=q$, which may be assumed by induction on $k$. So it remains to deduce (iii) from its simplification. For that purpose, define $Y$ as

$$
\begin{gathered}
Y:=\left\{\left(q, c^{(1)}, c^{(2)}, \ldots, c^{(k)}, b^{(1)}, b^{(2)}, \ldots, b^{(k)}\right) \in W \times\left(\mathbb{C}^{n}\right)^{2 k} \mid\right. \\
H\left(t c^{(i)}+q\right)=H\left(t c^{(i)}+p^{(i)}\right)=0 \text { and }\left(b^{(i)}\right)^{\mathrm{t}} c^{(i)}=1 \\
\text { for each } \left.i, \text { and } \operatorname{rk}\left(q\left|c^{(1)}\right| c^{(2)}|\cdots| c^{(k)}\right) \leq 2\right\}
\end{gathered}
$$

We can write $Y$ as a union of algebraic sets of the form

$$
\begin{aligned}
& \left\{\left(q, c^{(1)}, \ldots, c^{(k)}, b^{(1)}, \ldots, b^{(k)}\right) \in Y \mid c^{(i)} \in L_{p^{(i)}}\right. \\
& \text { for each } \left.i, \text { and } \operatorname{rk}\left(q\left|c^{(1)}\right| c^{(2)}|\cdots| c^{(k)}\right) \leq 2\right\}
\end{aligned}
$$


where $L_{p^{(i)}} \ni p^{(i)}$ is a GN-plane of $H$ for each $i$. This union is finite by assumption.

Let $f$ be the projection of $\mathbb{C}^{n+2 k n}$ onto its first $n$ coordinates. From the simplified version of (iii), it follows that the image of $\left.f\right|_{Y}$ contains all $q \in W$ which contains only finitely many GN-planes of $H$. On account of (ii), the image of $\left.f\right|_{Y}$ is not contained in a proper algebraic subset of $W$. Hence there exists an irreducible component $Z$ of $Y$ such that the image of $\left.f\right|_{Z}$ is not contained in a proper algebraic subset of $W$. From Mumford $(1999, \S 1.8$, Th. 3), it follows that the image of $\left.f\right|_{Z}$ contains an open subset of $W$.

Since $Y$ is a finite union of algebraic subsets of the form (3.1) and $Z$ is irreducible, we deduce that $Z$ is contained in an algebraic subset of the form (3.1). Take

$$
\begin{array}{r}
\tilde{Z}:=\left\{\left(q, c^{(1)}, c^{(2)}, \ldots, c^{(k)}, b^{(1)}, b^{(2)}, \ldots, b^{(k)}\right) \in Z \mid\right. \\
\left.b^{(i)} \text { is the complex conjugate of } c^{(i)} \text { for each } i\right\}
\end{array}
$$

By applying proper substitutions in $t$ and $y_{1}, y_{2}, \ldots, y_{k}$, we see that the image $\tilde{X}$ of $\left.f\right|_{\tilde{Z}}$ is the same as that of $\left.f\right|_{Z}$, so $\tilde{X}$ contains an open subset of $W$. From Lemma 3.1, it follows that $\tilde{X}=W$. Since $\tilde{X}$ is the image of the restriction of $f$ on an algebraic subset of the form (3.1), the unsimplified (iii) follows.

Definition 3.9 Let $X$ be any subset of $\mathbb{C}^{n}$. We say that $a \in \mathbb{C}^{n}$ is an apex of $X$ if $(1-\lambda) c+\lambda a \in X$ for all $\lambda \in \mathbb{C}$ and all $c \in X$.

We say that a $p \in \mathbb{C}^{n}$ is a projective apex of $X$ if $p \neq 0$ and $c+\lambda p \in X$ for all $\lambda \in \mathbb{C}$ and all $c \in X$.

If $X$ is the Zariski closure of the image of a map $H$, then we say that $a$ and $p$ as above are an image apex of $H$ and a projective image apex of $H$ respectively.
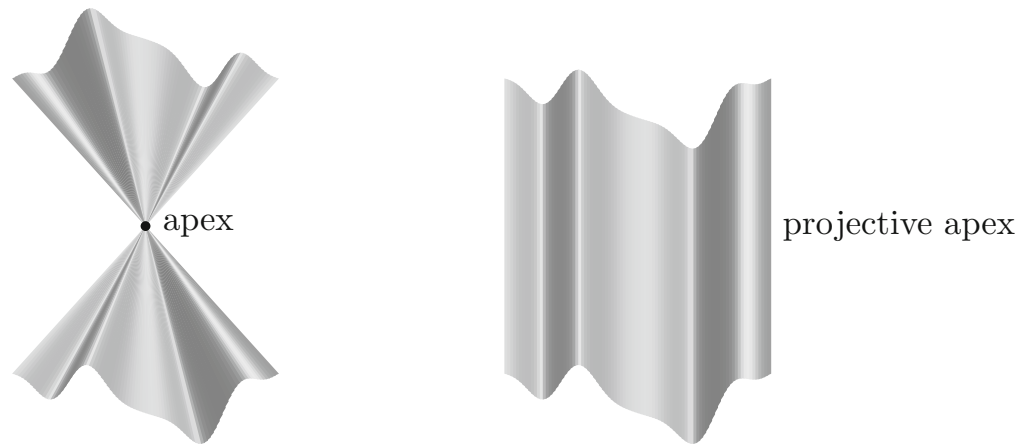

One may convince oneself that a projective apex is in fact an apex on the projective horizon.

If $X$ is a zero set of homogeneous polynomials, e.g. because $X$ is the Zariski closure of the image of a homogeneous map, then 0 is an apex of $X$. If 0 is an apex of $X$, then a projective image apex is the same as a nonzero apex. In that case, we will parenthesize the word projective. 
Corollary 3.10 Assume $x+H$ is a homogeneous quasi-translation over $\mathbb{C}$, such that $\mathrm{rk} \mathcal{J} H \leq(n+1) / 2$. Write $W$ for the Zariski closure of the image of $H$. Then for

(1) $\operatorname{dim} V(H)=\mathrm{rk} \mathcal{J} H \leq 3$ and $W$ has no nonzero (projective) apex;

(2) $\operatorname{dim} V(H)=\operatorname{rk} \mathcal{J} H$ and there is no nonzero $p \in W$ which contains infinitely many GN-planes of $H$ that are contained in $W$;

(3) There exists a $p \in W$ which is contained in only finitely many GN-planes of $H$, but there does not exist a nonzero $c \in V(H)$ which shares a GN-plane of $H$ with every $q \in W$;

(4) $\mathrm{rk} \mathcal{J} H \leq 1$ or $W$ is properly contained in the linear span of two GN-planes of $H$ which are contained in $W$;

(5) $W$ is a properly contained in a 4-dimensional linear subspace of $\mathbb{C}^{n}$ and rk $\mathcal{J} H \leq 3$;

we have (1) $\Rightarrow(2) \Rightarrow(3) \Rightarrow(4) \Rightarrow(5)$.

Proof From Lemma 3.2, it follows that $W$ is irreducible and that $\operatorname{rk} \mathcal{J} H=\operatorname{dim} W$.

$(1) \Rightarrow(2)$ Assume that $\operatorname{dim} V(H)=\operatorname{rk} \mathcal{J} H \leq 3$ and that (2) does not hold. Then there exists a nonzero $p \in W$ which contains infinitely many GN-planes of $H$ that are contained in $W$. Suppose that $W$ is the zero set of $g_{1}, g_{2}, \ldots, g_{m}$ and let

$Y=\left\{q \in W \mid g_{1}(p+t q)=g_{2}(p+t q)=\cdots=g_{m}(p+t q)=0\right\}$

Then $Y$ has an irreducible component $Z$ which contains infinitely many GN-planes of $H$. Hence $\operatorname{dim} Z \geq 3$. Since $Z \subseteq Y \subseteq W$ and $\operatorname{dim} W=\operatorname{rk} \mathcal{J} H \leq 3$, it follows from the irreducibility of $Z$ and $W$ that $Z=W$. So $p$ is a nonzero (projective) apex of $W$ and (1) does not hold.

(2) $\Rightarrow$ (3) Assume that $\operatorname{dim} V(H)=\operatorname{rk} \mathcal{J} H$ and that (3) does not hold. Since $\operatorname{dim} V(H)=\operatorname{rk} \mathcal{J} H$, it follows from Lemma 3.2 that $W$ is an irreducible component of $V(H)$, so the interior of $W$ as a closed subset of $V(H)$ is nonempty. Take $p$ in that interior and let $L_{p} \ni p$ be a GN-plane of $H$. Since $L_{p}$ is irreducible, $L_{p}$ is contained in an irreducible component of $V(H)$, which can only be $W$ because $W$ is the only irreducible component of $V(H)$ which contains $p$.

So if $p$ is contained in infinitely many GN-planes of $H$, then (2) cannot hold. Hence assume that $p$ is contained in only finitely many GN-planes of $H$. Since (3) does not hold, there exists a nonzero $c \in V(H)$ which shares a GN-plane of $H$ with every $q \in W$. Inductively, we can choose $p^{(i)}$ in the interior of $W$ outside $L_{p^{(1)}}, L_{p^{(2)}}, \ldots, L_{p^{(i-1)}}$ for $i=1,2,3, \ldots$, such that $c \in L_{p^{(i)}}$ for each $i$. As we have seen above, $L_{p^{(i)}} \subseteq W$ for each $i$, so $c$ is a counterexample to the claim of (2).

(3) $\Rightarrow$ (4) Assume that (3) is satisfied. From (ii) of Theorem 3.8, it follows that there exist a $p^{(1)} \in W$ and a $p^{(2)} \in W$ as in (iii) of Theorem 3.8. Take $L_{p^{(1)}}$ and $L_{p^{(2)}}$ as in (iii) of Theorem 3.8. Since there is no nonzero $c \in V(H)$ which shares a GN-plane of $H$ with every $q \in W, W$ cannot be equal to 
any linear span. Hence it suffices to show that $W$ is contained in the linear span of $L_{p^{(1)}}$ and $L_{p^{(2)}}$. In the case where $L_{p^{(1)}} \cap L_{p^{(2)}}=\{0\}$, this follows directly from (iii) of Theorem 3.8, so assume that there exist a nonzero $c \in L_{p^{(1)}} \cap L_{p^{(2)}}$. Let

$$
Y=\{q \in W \mid H(c+t q)=0\}
$$

From (3), it follows that $Y$ is a proper algebraic subset of $W$. Since $W$ irreducible and contained in the union of $Y$ and the linear span of $L_{p^{(1)}}$ and $L_{p^{(2)}}, W$ is contained in the linear span of $L_{p^{(1)}}$ and $L_{p^{(2)}}$.

(4) $\Rightarrow$ (5) Assume that (4) is satisfied. If $\mathrm{rk} \mathcal{J} H \leq 1$, then $W$ is a line through the origin on account of (i) of Theorem 3.6, which gives (5). So assume that $\mathrm{rk} \mathcal{J} H \geq 2$. Then $W$ is properly contained in a 4-dimensional linear subspace of $\mathbb{C}^{n}$ and hence rk $\mathcal{J} H=\operatorname{dim} W<4$.

Remark 3.11 Theorem 3.6 was obtained in Gordan and Nöther (1876, p. 565), but Gordan and Nöther proved additionally that $\mathcal{J} H \cdot H(y)=0$ if rk $\mathcal{J} H \leq 2$ and rk $\mathcal{J} H+\operatorname{dim} V(H) \leq n$. See de Bondt (2014, Th. 4.1) for properties that are equivalent to $\mathcal{J} H \cdot H(y)=0$.

The starting point of the distinction into cases 'Fall a)' and 'Fall b)' on Gordan and Nöther (1876, p. 565) is (i) of Theorem 3.8, but with the extra property that $L_{p}$ and $L_{q}$ are contained in $W$. Since $\operatorname{dim} V(H)=\operatorname{rk} \mathcal{J} H=\operatorname{dim} W$ in this situation, this extra property can indeed be obtained, namely by extending the genericity condition in the proof of (i) of Theorem 3.8 by that $p$ and $q$ are in the interior of $W$ as a closed subset of $V(H)$.

The case where $k=2$ of (iii) of Theorem 3.8 is obtained on Gordan and Nöther (1876, p. 566), and is used on the same page to prove the case where $n=5$ and rk $\mathcal{J} H=3$ of Corollary 3.10.

\section{Homogeneous singular Hessians in dimension 5}

Gordan and Nöther (1876) classified all homogeneous polynomials with singular Hessians in dimension 5 as follows.

Theorem 4.1 (Gordan and Nöther) Assume $h \in \mathbb{C}[x]$ is a homogeneous polynomial in dimension $n=5$. If $\operatorname{det} \mathcal{H} h=0$ and $h$ is not a polynomial in $n-1=4$ linear forms in $\mathbb{C}[x]$, then there exists an invertible matrix $T$ over $\mathbb{C}$ such that $h(T x)$ is of the form

$$
h(T x)=f\left(x_{1}, x_{2}, a_{1}\left(x_{1}, x_{2}\right) x_{3}+a_{2}\left(x_{1}, x_{2}\right) x_{4}+a_{3}\left(x_{1}, x_{2}\right) x_{5}\right)
$$

where $f$ and $a_{1}, a_{2}, a_{3}$ are polynomials over $\mathbb{C}$ in their arguments.

The proof that is given below uses results about homogeneous quasi-translations in dimension five and follows the approach of Gordan and Nöther more or less.

The following connection exists between singular Hessians and quasi-translations. 
Proposition 4.2 (Gordan and Nöther) Assume $h \in \mathbb{C}[x]$ such that $\operatorname{det} \mathcal{H} h=0$. Then there exists a nonzero $R \in \mathbb{C}[y]$ such that $R(\nabla h)=0$. For any such $R, x+H$ is a quasi-translation and $(\nabla h)(x+t H)=\nabla h$, where $H:=(\nabla R)(\nabla h)$, and $H \neq 0$ if $R$ has minimum degree. Furthermore, $h(x+t H)=h$ if $R^{*}(\nabla h)=0$ for every homogeneous part of $R^{*}$ of $R$.

Proof From Proposition 1.2.9 of either van den Essen (2000) or de Bondt (2009), it follows that the components of $\nabla h$ are algebraically dependent over $\mathbb{C}$, so $R$ indeed exists. By the chain rule,

$$
\mathcal{J} H \cdot H=\left.(\mathcal{H} R)\right|_{y=\nabla h} \cdot \mathcal{H} h \cdot H
$$

So if $\mathcal{H} h \cdot H=0$, then $x+H$ is a quasi-translation on account of (3) $\Rightarrow$ (1) of Proposition 2.2. Indeed, if we take the Jacobian of $R(\nabla h)=0$, we obtain

$$
\mathcal{J} 0=\mathcal{J}(R(\nabla h))=(\mathcal{J} R)_{y=\nabla h} \cdot \mathcal{H} h=H^{\mathrm{t}} \cdot \mathcal{H} h
$$

which gives $\mathcal{H} h \cdot H=0$, because $\mathcal{H} h$ is symmetric. Furthermore, (2.3) in Proposition 2.2 tells us that $(\nabla h)(x+t H)=0$.

If $R$ has minimum degree and $H_{i}=0$, then $\frac{\partial}{\partial y_{i}} R=0$ because $\left(\frac{\partial}{\partial y_{i}} R\right)(\nabla h)$ $=H_{i}=0$. Since $R \notin \mathbb{C}$, we see that $H \neq 0$ if $R$ has minimum degree.

Suppose that $R^{*}(\nabla h)=0$ for every homogeneous part $R^{*}$ of $R$. Let $E_{y}: \mathbb{C}[y]$ $\rightarrow \mathbb{C}[y]$ be the map which multiplies each term by its own degree in $y$. Then one can verify that $E_{y} R=y^{\mathrm{t}} \nabla R$, and that $E_{y} R$ is a linear combination of the homogeneous parts $R^{*}$ of $R$. So $\mathcal{J} h \cdot H=\left(y^{\mathrm{t}} \nabla R\right)_{y=\nabla h}=\left(E_{y} R\right)_{y=\nabla h}=0$. Hence $h(x+t H)=h$ on account of (2.3) in Proposition 2.2.

In order to prove Theorem 4.1, we need the classification of all homogeneous polynomials with singular Hessians in dimensions less than 5, which is as in Theorem 4.3 below. Our proof of Theorem 4.3 is somewhat different from that by Gordan and Nöther. A proof of Theorem 4.3 which is based on that by Gordan and Nöther can be found in Lossen (2004).

Theorem 4.3 (Gordan and Nöther) Assume $h \in \mathbb{C}[x]$ is a homogeneous polynomial in dimension $n \leq 4$. If $\operatorname{det} \mathcal{H} h=0$, then the components of $\nabla$ h are linearly dependent over $\mathbb{C}$.

Proof Suppose that the components of $\nabla h$ are linearly independent over $\mathbb{C}$. Then $\operatorname{deg} \nabla h \geq 1$ because $\operatorname{det} \mathcal{H} h=0$. Let $H=(\nabla R)(\nabla h)$ as in Proposition 4.2, such that $R$ has minimum degree. Then $H$ is a nonzero quasi-translation and $\operatorname{deg} H \geq$ 1 because $\operatorname{deg} R \geq 2$ and $\operatorname{deg} \nabla h \geq 1$. Furthermore, $H$ is homogeneous because $R$ and $\nabla h$ are homogeneous. From (2) $\Rightarrow$ (3) of Proposition 2.6, it follows that $r:=\operatorname{rk} \mathcal{J} H \leq \max \{n-2,1\} \leq 2$. Using (i) and (iii) of Theorem 3.6, we can deduce that $x+H$ has $n-r<n$ linear invariants.

Since $n-r<n$, there exists a nonzero $p \in \mathbb{C}^{n}$ which is a zero of all these $n-r$ linear invariants. From Proposition 1.2.9 of either van den Essen (2000) or de Bondt (2009), it follows that $\operatorname{trdeg}_{\mathbb{C}}(H)=r$. Hence the $n-r$ linear invariants of 
$x+H$ generate the ideal $(\tilde{R} \in \mathbb{C}[y] \mid \tilde{R}(H)=0)$ of $\mathbb{C}[y]$. Consequently, $p$ is a projective image apex of $H$. From Lemma 4.4 below, it follows that $\mathcal{J} h \cdot p=0$, so the components of $\nabla h$ are linearly dependent over $\mathbb{C}$ indeed.

Lemma 4.4 Let $h \in \mathbb{C}[x]$ and $R \in \mathbb{C}[y]$, such that $R^{*}(\nabla h)=0$ for every homogeneous part $R^{*}$ of $R$.

Then $\mathcal{J} h \cdot \mathcal{J} H=0$, where $H:=(\nabla R)(\nabla h)$. Furthermore, if $p$ is a projective image apex of $H$, then $\mathcal{J} h \cdot p=0$.

Proof From Proposition 4.2, it follows that $h(x+t H)=h$. By taking the Jacobian on both sides, we obtain

$$
\left.(\mathcal{J} h)\right|_{x=x+t H} \cdot\left(I_{n}+t \mathcal{J} H\right)=\mathcal{J} h
$$

From Proposition 4.2 again, it follows that $\left.(\mathcal{J} h)\right|_{x=x+t H}=\mathcal{J} h$, so $\mathcal{J} h \cdot t \mathcal{J} H=0$, which gives the first claim.

Suppose that $p$ is a projective image apex of $H$. Take $T \in \mathrm{GL}_{n}(\mathbb{C})$ such that the last column of $T$ equals $p$. Then $e_{n}$ is a projective image apex of $\tilde{H}:=$ $T^{-1} H$. So $\tilde{H}_{n}$ is algebraically independent of $\tilde{H}_{1}, \tilde{H}_{2}, \ldots, \tilde{H}_{n-1}$. Hence $\operatorname{trdeg} \mathbb{C} \mathbb{C}(\tilde{H})$ $=\operatorname{trdeg}_{\mathbb{C}} \mathbb{C}\left(\tilde{H}_{1}, \tilde{H}_{1}, \ldots, \tilde{H}_{n-1}\right)+1$. From proposition 1.2.9 of either van den Essen (2000) or de Bondt (2009), it follows that the last row of $\mathcal{J} \tilde{H}$ is independent of the rows above it.

But $\mathcal{J} h \cdot T \cdot \mathcal{J} \tilde{H}=\mathcal{J} h \cdot \mathcal{J} H=0$. Hence the rightmost entry of $\mathcal{J} h \cdot T$ is zero. So $\mathcal{J} h \cdot p=0$ indeed.

Theorem 4.1 is formulated as de Bondt and van de Essen (2004, Th. 3.6). The starting point of the proof of de Bondt and van de Essen (2004, Th. 3.6) is de Bondt and van de Essen (2004 Th. 2.1 iii)), which is not accompanied by a proof and comes down the following.

Theorem 4.5 (Gordan and Nöther) Assume $h \in \mathbb{C}[x]$ is a homogeneous polynomial in dimension $n=5$. Suppose that $R(\nabla h)=0$, such that $R$ has minimum degree. Then $R$ can be expressed as a polynomial in three linear forms over $y$.

Proof Notice that $R$ is homogeneous because $h$ is homogeneous and $R$ has minimum degree. We distinguish two cases.

- $R$ cannot be expressed as a polynomial in four linear forms over $y$. Then the components of $\nabla R$ are linearly independent over $\mathbb{C}$. Since $R$ has minimum degree, the components of $H:=(\nabla R)(\nabla h)$ are linearly independent over $\mathbb{C}$ as well. Write $H=g \tilde{H}$, where $g \in \mathbb{C}[x]$, such that $\operatorname{gcd}\left\{\tilde{H}_{1}, \tilde{H}_{2}, \ldots, \tilde{H}_{n}\right\}=1$. Since the components of $H$ and hence also $\tilde{H}$ are linearly independent over $\mathbb{C}$, we have $\operatorname{deg} \tilde{H} \geq 1$. On account of Proposition 2.3, rk $\mathcal{J} \tilde{H}=\operatorname{rk} \mathcal{J} H$.

Since the components of $\tilde{H}$ are linearly independent over $\mathbb{C}$, it follows from Theorem 3.6 that $3 \leq \operatorname{rk} \mathcal{J} \tilde{H} \leq \operatorname{dim} V(\tilde{H}) \leq n-2$, so $\operatorname{rk} \mathcal{J} \tilde{H}=\operatorname{dim} V(\tilde{H})=3$. From $(1) \Rightarrow(5)$ of Corollary 3.10 , it follows that $\tilde{H}$ has a projective image apex, say $p$. Then $f(\tilde{H})=0$ implies $f(\tilde{H}+t p)=0$ for every homogeneous $f \in \mathbb{C}[y]$. Hence $f(H)=0$ implies $f(H+\operatorname{tg} p)=0$ for every homogeneous $f \in \mathbb{C}[y]$. 
Since $H$ is homogeneous, we can substitute $t=g^{-1} t$ to deduce that $p$ is a projective image apex of $H$ as well.

From Lemma 4.4, it subsequently follows that $\mathcal{J} h \cdot p=0$. Hence the components of $\nabla h$ are linearly dependent over $\mathbb{C}$. Since $R$ has minimum degree, we conclude that $\operatorname{deg} R=1$, so $R$ is a linear form in $\mathbb{C}[y]$. Contradiction.

- $R$ can be expressed as a polynomial in four linear forms over $y$.

Then there is an $i \leq 5$ such that $y_{i}$ is not a linear combination of these four linear forms. Say that $i=5$. Then $R$ is of the form $\tilde{R}\left(y_{1}+c_{1} y_{5}, y_{2}+c_{2} y_{5}, y_{3}+\right.$ $\left.c_{3} y_{5}, y_{4}+c_{4} y_{5}\right)$, where $c_{i} \in \mathbb{C}$ for each $i$. Furthermore, $\tilde{R} \in \mathbb{C}\left[y_{1}, y_{2}, y_{3}, y_{4}\right]$ is homogeneous and $\tilde{R}(\nabla \tilde{h})=\tilde{R}(\nabla \hat{h})=0$, where

$$
\tilde{h}=\left.h\right|_{x_{5}=x_{5}+c_{1} x_{1}+c_{2} x_{2}+c_{3} x_{3}+c_{4} x_{4}} \text { and } \hat{h}=\left.\tilde{h}\right|_{x_{5}=1}
$$

Since $\tilde{h}$ is homogeneous, say of degree $d$, it follows that $\tilde{h}=x_{5}^{d} \hat{h}\left(x_{5}^{-1} x\right)$ and that $\nabla \tilde{h}$ and $x_{5}^{d-1}(\nabla \hat{h})\left(x_{5}^{-1} x\right)$ agree on the first 4 components. From this, we can deduce that $\tilde{R}$, as a homogeneous polynomial in $\mathbb{C}\left[y_{1}, y_{2}, y_{3}, y_{4}\right]$ such that $\tilde{R}(\nabla \hat{h})=0$, has minimum degree as well. From Theorem 4.6 below, we obtain that $\tilde{R}$ can be expressed as a polynomial in three linear forms in $\mathbb{C}\left[y_{1}, y_{2}, y_{3}, y_{4}\right]$. Hence $R$ can be expressed as a polynomial in three linear forms in $\mathbb{C}[y]$.

Theorem 4.6 Let $n=4$ and $h \in \mathbb{C}[x]$, not necessarily homogeneous. Suppose that $R \in \mathbb{C}[y]$ is homogeneous, such that $R(\nabla h)=0$. If $R$ has minimum degree, then $R$ can be expressed as a polynomial in three linear forms in $\mathbb{C}[y]$.

Proof Suppose that $R$ has minimum degree. Let $\bar{h}$ be the leading homogeneous part of $h$, and define $H:=(\nabla R)(\nabla h)$. From Proposition 4.2, it follows that $h(x+t H)=h$. By taking the leading coefficient with respect to $t$, we deduce that $\bar{h}(H)=0$.

Since $\bar{h}$ is homogeneous and $R(\nabla \bar{h})=0$, it follows from Theorem 4.3 that the components of $\nabla \bar{h}$ are linearly dependent over $\mathbb{C}$, say that $L(\nabla \bar{h})=0$ for some linear form $L \in \mathbb{C}[y]$. Assume first that $\mathrm{rk} \mathcal{H} \bar{h}=3$. Then the relations between the components of $\nabla \bar{h}$ form a prime ideal of height one, which is a principal ideal because $\mathbb{C}[y]$ is a unique factorization domain. Since $L$ is irreducible, $(L)$ must be that principal ideal, and $L \mid R$ because $R(\nabla \bar{h})=0$. Since $R$ has minimum degree, $R$ is irreducible, so $R$ is linear.

Assume next that $\mathrm{rk} \mathcal{H} \bar{h} \leq 2$. Since there exists a linear relation between the components of $\nabla \bar{h}$, there exists a $T \in \mathrm{GL}_{n}(\mathbb{C})$ such that the last component of $T^{\mathrm{t}} \nabla \bar{h}$ is zero. Hence the last component of $\nabla(\bar{h}(T x))=T^{\mathrm{t}}(\nabla \bar{h})(T x)$ is zero. So $\bar{h}(T x)$ $\in \mathbb{C}\left[x_{1}, x_{2}, x_{3}\right]$. Since $\mathcal{H}(\bar{h}(T x))=\left.T^{\mathrm{t}}(\mathcal{H} \bar{h})\right|_{x=T x} T$, we see that $\operatorname{rk} \mathcal{H}(\bar{h}(T x)) \leq 2$. It follows from Theorem 4.3 again that $\bar{h}(T x)$ can be expressed as a polynomial in two linear forms. Hence $\bar{h}=\bar{h}\left(T\left(T^{-1} x\right)\right)$ can be expressed as a polynomial in two linear forms as well.

Since $\bar{h}$ is homogeneous in addition, $\bar{h}$ decomposes into linear factors, and one of these factors is already a relation between $\mathrm{H}_{1}, \mathrm{H}_{2}, \mathrm{H}_{3}, \mathrm{H}_{4}$. So there exist a linear form $M \in \mathbb{C}[x]$ such that $M((\nabla R)(\nabla h))=M(H)=0$. Since $R$ has minimum degree, $M(\nabla R)=0$. On account of Example 1.2 in de Bondt and van den Essen (2004), $R$ can be expressed as a polynomial in three linear forms over $y$. 
Remark 4.7 The proof of the first case in the proof of Theorem 4.5 is different from that given in Gordan and Nöther (1876, p. 568), where the second claim of Lemma 4.4 is obtained by way of differentiation on the inverse of $H$. Since the inverse of $H$ is not a map, the above proof of this first case seems much easier. The proof of this first case as given in de Bondt (2009, Th. 5.3.7) is incorrect.

The proof of the second case in the proof of Theorem 4.5 comes from Gordan and Nöther (1876, p. 567). This seems a little odd, because Lemma 4.6 is about not necessarily homogeneous polynomials, which Gordan and Nöther did not consider in Gordan and Nöther (1876). But in spite of that, the proof of Lemma 4.6 comes from Gordan and Nöther (1876, p. 567) indeed.

On Gordan and Nöther (1876, p. 567), Gordan and Nöther additionally prove that rk $\mathcal{J} H \leq 2$, as follows. They assume that $H_{1}=H_{2}=0$ on account of Theorem 4.5 and Proposition 2.4, and show the first claim of Lemma 4.4 that $\mathcal{J} h \cdot \mathcal{J} H=0$, to conclude that either $h \in \mathbb{C}\left[x_{1}, x_{2}\right]$ or that the rows of $\mathcal{J}\left(H_{3}, H_{4}, H_{5}\right)$ are dependent. In both cases, rk $\mathcal{J} H \leq 2$ indeed, because $H_{i} \in \mathbb{C}\left[\frac{\partial}{\partial x_{1}} h, \frac{\partial}{\partial x_{2}} h\right]$ for all $i$ in the first case, so that the row space of $\mathcal{J} H$ is generated by $\mathcal{J}\left(\frac{\partial}{\partial x_{1}} h\right)$ and $\mathcal{J}\left(\frac{\partial}{\partial x_{2}} h\right)$.

Unlike Gordan and Nöther, we do not need to show that rk $\mathcal{J} H \leq 2$ here, because for the techniques in de Bondt and van den Essen (2004), linear dependences between the components of $H$ are the only thing that matters. But the result of Gordan and Nöther can be used to fix the gap in Watanabe (2014), which is caused by the incorrect (Watanabe 2014, Lm. 5.2), and a gap on the same point in Franchetta (1954).

\section{Homogeneous 5-dimensional quasi-translations of 'Fall b)'}

In this section, we study homogeneous quasi-translations in dimension 5 which corresponds to 'Fall b)' in Gordan and Nöther (1876, §8). In Corollary 5.2, we will show that homogeneous quasi-translations in dimension 5 which are not of this type always have a linear invariant.

Theorem 5.1 Assume $x+H$ is a homogeneous quasi-translation in dimension 5 over $\mathbb{C}$, such that $\operatorname{gcd}\left\{H_{1}, H_{2}, H_{3}, H_{4}, H_{5}\right\}=1$ and $H_{5}$ is algebraically independent over $\mathbb{C}$ of $\mathrm{H}_{1}, \mathrm{H}_{2}, \mathrm{H}_{3}, \mathrm{H}_{4}$.

If $x+H$ does not have two independent linear invariants, then $\mathrm{rk} \mathcal{J} H$ $=\operatorname{dim} V(H)=3$ and the following holds.

(i) $H$ is of the form

$$
H=\left(g h_{1}(p, q), g h_{2}(p, q), g h_{3}(p, q), g h_{4}(p, q), H_{5}\right)
$$

where $g \in \mathbb{C}[x], h \in \mathbb{C}\left[y_{1}, y_{2}\right]^{4}$ and $(p, q) \in \mathbb{C}[x]^{2}$ are homogeneous, and $\operatorname{gcd}\{p, q\}=1$.

(ii) $g, p, q$ are invariants of $x+H$, and $g \in \mathbb{C}\left[x_{1}, x_{2}, x_{3}, x_{4}\right]$.

(iii) $\operatorname{deg}_{x_{5}} H_{5} \leq \operatorname{deg}_{x_{5}}\left(H_{1}, H_{2}, H_{3}, H_{4}\right)$ and there exists a linear combination over $\mathbb{C}$ of $p$ and $q$ whose degree with respect to $x_{5}$ is less than $\max \left\{\operatorname{deg}_{x_{5}} p, \operatorname{deg}_{x_{5}} q\right\}$. 
(iv) There exists an invariant $a \in \mathbb{C}[x]$ of degree at most 1 of $x+H$, such that every invariant of $x+H$ which can be expressed as a polynomial in four linear forms in $\mathbb{C}[x]$ is contained in $\mathbb{C}[a]$.

(v) If $H$ has no linear invariants at all, then $g \in \mathbb{C}$.

Proof From (ii) of Theorem 3.6, it follows that $2 \leq \mathrm{rk} \mathcal{J} H \leq \operatorname{dim} V(H) \leq 3$. Assume that $x+H$ does not have two independent linear invariants. From (iii) of Theorem 3.6, it follows that rk $\mathcal{J} H \neq 2$, so rk $\mathcal{J} H=\operatorname{dim} V(H)=3$.

(i) Since $H_{5}$ is algebraically independent over $\mathbb{C}$ of $H_{1}, H_{2}, H_{3}, H_{4}$, it follows from rk $\mathcal{J} H=3$ and Proposition 1.2.9 of either van den Essen (2000) or de Bondt (2009) that rk $\mathcal{J}\left(H_{1}, H_{2}, H_{3}, H_{4}\right)=2$. Using (de Bondt and van den Essen 2005 , Th. 2.2) (see also de Bondt 2009, Th. 4.3.1), we see that $H$ is of the given form.

(ii) Take $i \leq 4$ such that $H_{i} \neq 0$. Then $g \cdot h_{i}(p, q)=H_{i}$ and on account of Proposition 2.2,

$$
\operatorname{deg}_{t} g(x+t H)+\operatorname{deg}_{t} h_{i}(p(x+t H), q(x+t H))=\operatorname{deg}_{t} H_{i}(x+t H)=0
$$

whence $\operatorname{deg}_{t} g(x+t H)=0$ and $g$ is an invariant of $x+H$. Similarly, any linear form in $p$ and $q$ that divides $H_{i}$ is an invariant of $x+H$ as well. If there is at most one independent linear form in $p$ and $q$ that divides $H_{i}$ for any $i \leq 4$ such that $H_{i} \neq 0$, then $\operatorname{deg} g=\operatorname{deg} H$ and $x+H$ has three independent linear invariants, which is a contradiction. Hence there are two independent linear forms in $p$ and $q$ that are invariants of $x+H$. Since $p$ and $q$ are in turn linear forms in these invariants, $p$ and $q$ are invariants of $x+H$ themselves.

Since $g$ is an invariant of $x+H$, it follows from (2.3) in Proposition 2.2 that $\mathcal{J} g \cdot H=0$. Hence

$$
g \mid \mathcal{J} g \cdot H-g \sum_{i=1}^{4} h_{i}(p, q) \frac{\partial}{\partial x_{i}} g=H_{5} \frac{\partial}{\partial x_{5}} g
$$

Now $\frac{\partial}{\partial x_{5}} g \neq 0$ contradicts the assumption that $\operatorname{gcd}\left\{g, H_{5}\right\} \quad \mid \operatorname{gcd}\left\{H_{1}\right.$, $\left.H_{2}, H_{3}, H_{4}, H_{5}\right\}=1$. Thus $g \in \mathbb{C}\left[x_{1}, x_{2}, x_{3}, x_{4}\right]$.

(iii) Let $r$ be the degree with respect to $x_{5}$ of $\left(H_{1}, H_{2}, H_{3}, H_{4}\right)$. If the degree with respect to $x_{5}$ of $H_{5}$ is larger than $r+1$, then $\operatorname{deg}_{x_{5}} \mathcal{J} H_{5} \cdot H=\operatorname{deg}_{x_{5}}\left(\frac{\partial}{\partial x_{5}} H_{5}\right) \cdot H_{5}>$ $2 r+1$, which contradicts (3) $\Rightarrow$ (1) of Proposition 2.2. Take for $\bar{H}_{i}$ all terms of degree $r$ with respect to $x_{5}$ of $H_{i}$ if $i \leq 4$, and for $\bar{H}_{5}$ all terms of degree $r+1$ with respect to $x_{5}$ of $H_{5}$.

Then the part of degree $2 r$ with respect to $x_{5}$ of $\mathcal{J}\left(H_{1}, H_{2}, H_{3}, H_{4}\right) \cdot H$ equals $\mathcal{J}\left(\bar{H}_{1}, \bar{H}_{2}, \bar{H}_{3}, \bar{H}_{4}\right) \cdot \bar{H}$ and the part of degree $2 r+1$ of $\mathcal{J} H_{5} \cdot H$ equals $\mathcal{J} \bar{H}_{5} \cdot \bar{H}$. Since $\mathcal{J} H_{i} \cdot H=0$ for all $i$ on account of (1) $\Rightarrow$ (3) of Proposition 2.2, we have $\mathcal{J} \bar{H} \cdot \bar{H}=0$.

On account of $(3) \Rightarrow$ (2) of Proposition 2.2, $\operatorname{deg}_{t} \bar{H}_{5}(x+t \bar{H})=0$. Since $x_{5} \mid \bar{H}_{5}$, $\operatorname{deg}_{t}(x+t \bar{H})_{5}=0$ as well. Hence $\bar{H}_{5}=0$ and $\operatorname{deg}_{x_{5}} H_{5} \leq r=\operatorname{deg}_{x_{5}}\left(H_{1}\right.$, $\left.H_{2}, H_{3}, H_{4}\right)$. By taking leading parts with respect to $x_{5}$, we see that for homogeneous and hence any $R \in \mathbb{C}\left[y_{1}, y_{2}, y_{3}, y_{4}\right], R\left(H_{1}, H_{2}, H_{3}, H_{4}\right)=0$ implies 
$R\left(\bar{H}_{1}, \bar{H}_{2}, \bar{H}_{3}, \bar{H}_{4}\right)=0$. It follows from Proposition 1.2.9 of either van den Essen (2000) or de Bondt (2009) that

$$
\begin{aligned}
\operatorname{rk} \mathcal{J}\left(x_{5}^{-r} \bar{H}\right) & =\operatorname{trdeg}_{\mathbb{C}} \mathbb{C}\left(x_{5}^{-r} \bar{H}_{1}, x_{5}^{-r} \bar{H}_{2}, x_{5}^{-r} \bar{H}_{3}, x_{5}^{-r} \bar{H}_{4}\right) \\
& \leq \operatorname{trdeg}_{\mathbb{C}} \mathbb{C}\left(H_{1}, H_{2}, H_{3}, H_{4}\right)=\operatorname{rk} \mathcal{J}\left(H_{1}, H_{2}, H_{3}, H_{4}\right)=2
\end{aligned}
$$

Since $x_{5}^{-r} \bar{H}_{5}=0$ and $x_{5}^{-r} \bar{H}_{i} \in \mathbb{C}\left[x_{1}, x_{2}, x_{3}, x_{4}\right]$, we deduce that $x+\bar{H}$ can be regarded as a quasi-translation in dimension four (over its first four coordinates). By (i) and (iii) of Theorem 3.6, there are two independent linear forms $l_{1}$ and $l_{2}$ in $x_{1}, x_{2}, x_{3}, x_{4}$ such that $l_{1}\left(x_{5}^{-r} \bar{H}\right)=l_{2}\left(x_{5}^{-r} \bar{H}\right)=0$. So $\left.l_{1}(\bar{H})=l_{2} \bar{H}\right)=0$.

Suppose that the leading parts of $p$ and $q$ with respect to $x_{5}$ are independent and of the same degree with respect to $x_{5}$. Since $\left(\bar{H}_{1}, \bar{H}_{2}, \bar{H}_{3}, \bar{H}_{4}\right)$ is the leading part of $\left(H_{1}, H_{2}, H_{3}, H_{4}\right)$ with respect to $x_{5}$, it follows that $\left(\bar{H}_{1}, \bar{H}_{2}, \bar{H}_{3}, \bar{H}_{4}\right)=h(\bar{p}, \bar{q})$, where $\bar{p}$ and $\bar{q}$ are the leading parts of $p$ and $q$ respectively with respect to $x_{5}$. By assumption, $\bar{p}$ and $\bar{q}$ are independent, so we can deduce from $l_{1}(\bar{H})=l_{2}(\bar{H})=0$ that $l_{1}(h)=l_{2}(h)=0$ and hence also $l_{1}(H)=l_{2}(H)=0$. Contradiction, thus the leading parts of $p$ and $q$ with respect to $x_{5}$ are dependent or have different degrees with respect to $x_{5}$, as desired.

(iv) Take for $a$ the linear invariant of $x+H$, if it has any, and take $a=1$ otherwise. Let $f$ be a non-constant invariant of $x+H$ which can be expressed in four linear forms. We distinguish two cases.

- $f \in \mathbb{C}\left[x_{1}, x_{2}, x_{3}, x_{4}\right]$.

On account of (iii) above, we can obtain that $\operatorname{deg}_{x_{5}} p<\operatorname{deg}_{x_{5}} q$, namely by replacing $p$ and $q$ by linear combinations of $p$ and $q$, and adapting $h$ accordingly. If we replace $H$ by $T^{-1} H(T x)$ and $(f, p, q)$ by $(f(T x), p(T x), q(T x))$ for some $T \in \mathrm{GL}_{5}(\mathbb{C})$ such that the last column of $T$ is equal to the fifth unit vector, the form of $H$ does not change and neither do $\operatorname{deg}_{x_{5}} f, \operatorname{deg}_{x_{5}} p$ and $\operatorname{deg}_{x_{5}} q$. By choosing $T$ appropriate, we can obtain $-\infty \leq \operatorname{deg}_{y_{2}} h_{1}<$ $\operatorname{deg}_{y_{2}} h_{2}<\operatorname{deg}_{y_{2}} h_{3}<\operatorname{deg}_{y_{2}} h_{4}$.

On account of (2.3) in Proposition 2.2, $\mathcal{J} f \cdot H=0$. By looking at the leading coefficient with respect to $x_{5}$ in $\mathcal{J} f \cdot H$, we can successively deduce that $\frac{\partial}{\partial x_{4}} f=0, \frac{\partial}{\partial x_{3}} f=0, \frac{\partial}{\partial x_{2}} f=0$, and $H_{1}=0$. Hence $f$ is a polynomial over $\mathbb{C}$ in the invariant $x_{1}$ of $x+H$, and $f$ was a polynomial over $\mathbb{C}$ in the invariant $\left(T^{-1}\right)_{1} x$ of $x+H$ before replacing $H$ by $T^{-1} H(T x)$. Since $x+H$ does not have two independent linear invariants, we see that $f \in \mathbb{C}[a]$.

- $f \notin \mathbb{C}\left[x_{1}, x_{2}, x_{3}, x_{4}\right]$.

There exists a $T \in \mathrm{GL}_{5}(\mathbb{C})$ such that $f(T x) \in \mathbb{C}\left[x_{1}, x_{2}, x_{3}, x_{5}\right]$ and last column of $T$ is equal to the fifth unit vector. Just as above, we replace $H$ by $T^{-1} H(T x)$ and $(f, p, q)$ by $(f(T x), p(T x), q(T x))$. So we may assume that $f \in \mathbb{C}\left[x_{1}, x_{2}, x_{3}, x_{5}\right]$. From (2.3), it follows that $\mathcal{J} f \cdot H=0$ and that any homogeneous part of $f$ is an invariant of $x+H$ as well, so we may assume that $f$ is homogeneous.

Since $x+H$ has at most one linear invariant, we can use techniques in the proof of (i) of Theorem 3.6 to show that $\mathrm{rk} \mathcal{J}\left(H_{1}, H_{2}, H_{3}\right)=2$. Hence the ideal $\mathfrak{b}:=\left(R \in \mathbb{C}\left[y_{1}, y_{2}, y_{3}\right] \mid R\left(H_{1}, H_{2}, H_{3}\right)=0\right)$ has height 1 , and since 
$\mathbb{C}[y]$ is a unique factorization domain, $\mathfrak{b}$ is principal. Say that $R$ is a generator of $\mathfrak{b}$.

By looking at the leading homogeneous part of $f(x+H)=f$, we see that $f(H)=0$. Since $H_{5}$ is algebraically independent of $H_{1}, H_{2}, H_{3}$, we deduce that $R\left(x_{1}, x_{2}, x_{3}\right) \mid f$. From (2.3), it follows $f(x+t H)=f$, from which we can deduce that every factor of $f$ is an invariant of $x+H$. The case $f \in \mathbb{C}\left[x_{1}, x_{2}, x_{3}, x_{4}\right]$ above tells us that $R\left(x_{1}, x_{2}, x_{3}\right) \in \mathbb{C}[a]$, and $f / R\left(x_{1}, x_{2}, x_{3}\right) \in \mathbb{C}[a]$ follows by induction on the degree of $f$.

(v) From (ii), it follows that $g \in \mathbb{C}\left[x_{1}, x_{2}, x_{3}, x_{4}\right]$. On account of (iv), $g \in \mathbb{C}[a]$, where $a$ is as in (iv). If $H$ has no linear invariant, then $\operatorname{deg} a=0$. Hence $g \in$ $\mathbb{C}[a]=\mathbb{C}$ if $H$ has no linear invariant.

Corollary 5.2 Assume $x+H$ is a homogeneous quasi-translation over $\mathbb{C}$ in dimension 5 without linear invariants. Then $\operatorname{deg} H \geq 12$. More precisely, there exists a $T \in$ $\mathrm{GL}_{5}(\mathbb{C})$ such that $T^{-1} H(T x)$ is of the form

$$
T^{-1} H(T x)=g \cdot\left(h_{1}(p, q), h_{2}(p, q), h_{3}(p, q), h_{4}(p, q), f\right)
$$

where $h$ is homogeneous of degree at least 3 and $(p, q)$ is homogeneous of degree at least 4.

Proof On account of Proposition 2.3, we may assume that $\operatorname{gcd}\left\{H_{1}, H_{2}, H_{3}, H_{4}\right.$, $\left.H_{5}\right\}=1$. From (ii) of Theorem 3.6, it follows that $\operatorname{dim} V(H) \leq 3$. From (i) and (iii) of Theorem 3.6, it follows that rk $\mathcal{J} H \geq 3$. Using (2) $\Rightarrow$ (3) of Proposition 2.6, we can deduce that $\operatorname{dim} V(H)=\operatorname{rk} \mathcal{J} H=3$. From $(1) \Rightarrow(5)$ of Corollary 3.10, we obtain that $H$ has a nonzero (projective) image apex. From Proposition 2.4, it follows that we may assume that $e_{5}$ is a (projective) image apex.

From (i), (ii) and (v) of Theorem 5.1, it follows that there are invariants $p$ and $q$ of $x+H$, such that $H$ is of the form

$$
H=\left(h_{1}(p, q), h_{2}(p, q), h_{3}(p, q), h_{4}(p, q), H_{5}\right)
$$

such that $h$ and $(p, q)$ are homogeneous. Furthermore, it follows from (iii) and (iv) of Theorem 5.1 that we may assume that $\operatorname{deg}_{x_{5}} q>\operatorname{deg}_{x_{5}} p$ and $\operatorname{deg}_{x_{5}} p>0$ respectively.

On account of (2.3) in Proposition 2.2, $q(x+t H)=q(x)$, and looking at the leading coefficient with respect to $t$ gives $q(H)=0$. Since $e_{5}$ is a projective apex of $H$, we even have $q\left(H_{1}, H_{2}, H_{3}, H_{4}, H_{5}+t\right)=0$. Hence $\operatorname{deg}_{x_{5}} q \leq \operatorname{deg} q-1$ and in case of equality, looking at the leading coefficient with respect to $t$ in $q\left(x_{1}, x_{2}, x_{3}, x_{4}, t\right)$ gives a linear form $l_{1}$ such that $l_{1}\left(H_{1}, H_{2}, H_{3}, H_{4}\right)$, which contradicts that $x+H$ has no linear invariants. Thus $\operatorname{deg}_{x_{5}} q \leq \operatorname{deg} q-2$. If we combine this with the conclusion of the previous paragraph, then we obtain

$$
0<\operatorname{deg}_{x_{5}} p<\operatorname{deg}_{x_{5}} q \leq \operatorname{deg} q-2
$$

So $\operatorname{deg}(p, q) \geq \operatorname{deg} q \geq \operatorname{deg}_{x_{5}} q+2 \geq 4$. 
If $\operatorname{deg} h<3$, then there exists a linear form $l_{2} \in \mathbb{C}\left[x_{1}, x_{2}, x_{3}, x_{4}\right]$ such that $l_{2}(h)=0$ and hence also $l_{2}\left(H_{1}, H_{2}, H_{3}, H_{4}\right)=0$, which contradicts that $x+H$ has no linear invariants. Hence $\operatorname{deg} h \geq 3$.

The following theorem has been proved in Liu (2011) as well. The proof that is given below is somewhat less computational than that in Liu (2011).

Theorem 5.3 Assume $x+H$ is a homogeneous quasi-translation over $\mathbb{C}$ in dimension 5 without linear invariants. Then $\operatorname{deg} H \geq 15$. More precisely, $\operatorname{deg}(p, q) \geq 5$, where $p$ and $q$ are as in Corollary 5.2.

Proof Just like in the proof of Corollary 5.2, we may assume that $H$ is as in (5.1) such that $h$ is homogeneous of degree at least 3 and $(p, q)$ is homogeneous such that (5.2) is satisfied. If $\operatorname{deg} q \geq 5$, then $\operatorname{deg} H \geq 5 \operatorname{deg} h \geq 15$ indeed, because $(p, q)$ is homogeneous. Hence assume that $\operatorname{deg} q \leq 4$. We shall derive a contradiction.

(i) From (iv) of Theorem 5.1, it follows that $\operatorname{deg}_{x_{4}} p \geq 1$ and $\operatorname{deg}_{x_{4}} q \geq 1$. From (5.2), we deduce that $\operatorname{deg}_{x_{5}} p=1, \operatorname{deg}_{x_{5}} q=2$ and $\operatorname{deg} q=4$. Assume without loss of generality that $\operatorname{deg}_{y_{2}} h_{4}>\operatorname{deg}_{y_{2}} h_{3}>\operatorname{deg}_{y_{2}} h_{2}>\operatorname{deg}_{y_{2}} h_{1}$. Then $\operatorname{deg}_{x_{5}}$ $H_{4}>\operatorname{deg}_{x_{5}} H_{3}>\operatorname{deg}_{x_{5}} H_{2}>\operatorname{deg}_{x_{5}} H_{1}$.

Let $r$ be the leading coefficient with respect to $x_{5}$ of $q$. On account of (2.3) in Proposition 2.2, $\mathcal{J} q \cdot H=0$. By looking at the leading coefficient with respect to $x_{5}$ of $\mathcal{J} q \cdot H=0$, we deduce from (iii) of Theorem 5.1 that $r \in$ $\mathbb{C}\left[x_{1}, x_{2}, x_{3}\right]$. Since $q\left(H_{1}, H_{2}, H_{3}, H_{4}, t\right)=0, r\left(H_{1}, H_{2}, H_{3}\right)=0$ as well. By looking at the leading coefficient with respect to $x_{5}$ in $r\left(H_{1}, H_{2}, H_{3}\right)$, we see that the coefficients of $x_{3}^{2}$ and $x_{2} x_{3}$ of $r$ are zero. Hence $r$ is of the form $r=\left(\lambda_{1} x_{1}+\lambda_{2} x_{2}+\lambda_{3} x_{3}\right) x_{1}-\lambda_{4}^{2} x_{2}^{2}$, where $\lambda_{i} \in \mathbb{C}$ for all $i$. Since $r$ is irreducible, we have $\lambda_{3} \lambda_{4} \neq 0$.

(ii) We show that for invariants $f$ of $x+H$, we have $\operatorname{deg}_{x_{4}, x_{5}} f=\operatorname{deg}_{x_{5}} f$. Let $f$ be an invariant of $x+H$. From (2.3) in Proposition 2.2, it follows that $f(x+t H)=0$. Let $\bar{f}$ be the leading part of $f$ with respect to $\left(x_{4}, x_{5}\right)$ and suppose that $\operatorname{deg}_{x_{4}, x_{5}} f$ $>\operatorname{deg}_{x_{5}} f$. Then $x_{4} \mid \bar{f}$, say that $x_{4}^{v} \mid \bar{f}$ and $x_{4}^{v+1} \nmid \bar{f}$. On account of (iii) of Theorem 5.1, $\operatorname{deg}_{x_{5}} H_{4} \geq \operatorname{deg}_{x_{5}} H_{5}$ and $\operatorname{deg}_{x_{5}} H_{4}>\operatorname{deg}_{x_{5}} H_{i}$ for all $i \leq 3$. So $\operatorname{deg}_{x_{5}} H_{4} \geq \operatorname{deg}_{x_{5}} H_{i}-\operatorname{deg}_{x_{5}} x_{i}-1$ for all $i \neq 4$.

If we change a factor $x_{i}$ in a product into $t H_{i}$, the degree with respect to $x_{5}$ of that product will increase $\operatorname{deg}_{x_{5}} t H_{4}-\operatorname{deg}_{x_{5}} x_{4}=\operatorname{deg}_{x_{5}} t H_{4}$ if $i=4$ and $\operatorname{deg}_{x_{5}} t H_{i}-\operatorname{deg}_{x_{5}} x_{i} \leq \operatorname{deg}_{x_{5}} t H_{4}-1$ if $i \neq 4$. Having to do such a change $v$ times, starting with a term $u \in \mathbb{C}[x]$, we deduce from $\operatorname{deg}_{x_{5}} u=\operatorname{deg}_{x_{4}, x_{5}} u-\operatorname{deg}_{x_{4}} u$ for terms $u \in \mathbb{C}[x]$ that for any term and hence any polynomial $u \in \mathbb{C}[x]$, the coefficient of $t^{v}$ of $u(x+t H)$ has degree at most

$$
\begin{aligned}
b(u):= & \operatorname{deg}_{x_{4}, x_{5}} u-\operatorname{deg}_{x_{4}} u+\operatorname{deg}_{x_{4}} u \cdot \operatorname{deg}_{x_{5}} t H_{4} \\
& +\left(v-\operatorname{deg}_{x_{4}} u\right) \cdot\left(\operatorname{deg}_{x_{5}} t H_{4}-1\right)+ \\
= & v \cdot\left(\operatorname{deg}_{x_{5}} t H_{4}-1\right)+\operatorname{deg}_{x_{4}, x_{5}} u
\end{aligned}
$$


with respect to $x_{5}$. Since $b(u)$ is affinely linear in $\operatorname{deg}_{x_{4}, x_{5}} u$ as a function on terms $u \in \mathbb{C}[x]$, the part of degree $b(f)$ with respect to $x_{5}$ of the coefficient of $t^{v}$ of $f(x+t H)$ is equal to that of $\bar{f}(x+t H)$.

The part of degree $v$ with respect to $t$ of $\bar{f}\left(x_{1}, x_{2}, x_{3}, x_{4}+t H_{4}, x_{5}\right)$ equals $\left(t H_{4}\right)^{v} \frac{1}{v !} \frac{\partial^{v}}{\partial x_{4}^{v}} \bar{f}$. By definition of $v$,

$$
\begin{aligned}
\operatorname{deg}_{x_{5}}\left(\left(t H_{4}\right)^{v} \frac{1}{v !} \frac{\partial^{v}}{\partial x_{4}^{v}} \bar{f}\right) & =v \cdot \operatorname{deg}_{x_{5}} t H_{4}+\operatorname{deg}_{x_{5}} \frac{\partial^{v}}{\partial x_{4}^{v}} \bar{f} \\
& =v \cdot \operatorname{deg}_{x_{5}} t H_{4}-v+\operatorname{deg}_{x_{4}, x_{5}} \bar{f} \\
& =b(\bar{f})=b(f)
\end{aligned}
$$

so the part of degree $b(f)$ with respect to $x_{5}$ of the coefficient of $t^{v}$ of $\bar{f}\left(x_{1}, x_{2}, x_{3}, x_{4}+t H_{4}, x_{5}\right)$ is nonzero. Furthermore, we can deduce from $\operatorname{deg}_{x_{5}} t H_{4}-\operatorname{deg}_{x_{5}} x_{4}=\operatorname{deg}_{x_{5}} t H_{4}>\operatorname{deg}_{x_{5}} t H_{i}-\operatorname{deg}_{x_{5}} x_{i}$ for all $i \neq 4$ and $\operatorname{deg}_{x_{5}} \frac{\partial^{v}}{\partial x_{4}^{v}} \bar{f}=\operatorname{deg}_{x_{5}} \bar{f}$ that the degree with respect to $x_{5}$ of $\bar{f}(x+t H)$ $-\bar{f}\left(x_{1}, x_{2}, x_{3}, x_{4}+t H_{4}, x_{5}\right)$ is less than $b(f)$. Hence

$\operatorname{deg}_{x_{5}} f(x+t H)=\operatorname{deg}_{x_{5}} \bar{f}(x+t H)=\operatorname{deg}_{x_{5}} \bar{f}\left(x_{1}, x_{2}, x_{3}, x_{4}+t H_{4}, x_{5}\right)=b(f)$

But the coefficient of $t^{v}$ of $f(x+t H)$ is zero, so $v=0$. Hence $\operatorname{deg}_{x_{4}, x_{5}} f=$ $\operatorname{deg}_{x_{5}} f$ for invariants $f$ of $x+H$.

Since $p$ and $q$ are invariants of $x+H$, and $\operatorname{deg}_{x_{5}} p=1$ and $\operatorname{deg}_{x_{5}} q=2$, we have $\operatorname{deg}_{x_{4}, x_{5}} p=1$ and $\operatorname{deg}_{x_{4}, x_{5}} q=2$.

(ii) Let $\bar{H}_{i}$ be the part of $H_{i}$ that has degree $2 \operatorname{deg} h-1$ with respect to $\left(x_{4}, x_{5}\right)$, for $i=1,2,3$, and $\bar{H}_{j}$ the part of $H_{j}$ that has degree $2 \mathrm{deg} h$ with respect to $\left(x_{4}, x_{5}\right)$, for $j=4,5$. Then the part of degree $4 \operatorname{deg} h-2$ with respect to $\left(x_{4}, x_{5}\right)$ of $\mathcal{J}\left(H_{1}, H_{2}, H_{3}, H_{4}\right) \cdot H$ equals $\mathcal{J}\left(\bar{H}_{1}, \bar{H}_{2}, \bar{H}_{3}, \bar{H}_{4}\right) \cdot \bar{H}$ and the part of degree $4 \mathrm{deg} h-1$ with respect to $\left(x_{4}, x_{5}\right)$ of $\mathcal{J} H_{5} \cdot H$ equals $\mathcal{J} H_{5} \cdot H$. Since $\mathcal{J} H \cdot H=0$ on account of $(1) \Rightarrow$ (3) of Proposition 2.2, we have $\mathcal{J} \bar{H} \cdot \bar{H}=0$.

On account of (iii), $\operatorname{deg}_{x_{5}} H_{i}=\operatorname{deg}_{x_{4}, x_{5}} H_{i}$ for all $i$. Consequently, $\bar{H}_{1}=\bar{H}_{2}=0$ and $\bar{H}_{3}$ and $\left(\bar{H}_{4}, \bar{H}_{5}\right)$ are homogeneous with respect to $\left(x_{4}, x_{5}\right)$. We shall show that $\bar{H}_{5}$ is linearly dependent over $\mathbb{C}$ of $\bar{H}_{4}$, distinguishing the cases $\bar{H}_{3}=0$ and $\bar{H}_{3} \neq 0$.

Assume first that $\bar{H}_{3}=0$. From (2.4), it follows that $\mathcal{J} \bar{H}$ is nilpotent. Since $\bar{H}_{1}=\bar{H}_{2}=\bar{H}_{3}=0, \mathcal{J}_{x_{4}, x_{5}}\left(\bar{H}_{4}, \bar{H}_{5}\right)$ is nilpotent as well. From van den Essen (2000, Th. 7.2.25) (see also van den Essen and Hubbers 2000), we obtain that

$$
\left(\bar{H}_{4}, \bar{H}_{5}\right)=\left(b c\left(a x_{4}-b x_{5}\right)^{2 \operatorname{deg} h}, a c\left(a x_{4}-b x_{5}\right)^{2 \operatorname{deg} h}\right)
$$

Since $\operatorname{deg}\left(\bar{H}_{4}, \bar{H}_{5}\right)=2 \cdot 2 \mathrm{deg} h$, this is only possible if $a$ and $b$ are constant. Hence $\bar{H}_{5}=b^{-1} a \bar{H}_{4}$ is linearly dependent over $\mathbb{C}$ of $\bar{H}_{4}$.

Assume next that $\bar{H}_{3} \neq 0$. Let $\bar{q}$ be the leading and quadratic part of $q$ with respect to $\left(x_{4}, x_{5}\right)$. Then $\bar{q} \mid \bar{H}_{4}$, so $\operatorname{deg}_{t} \bar{q}(x+t \bar{H}) \leq \operatorname{deg}_{t} \bar{H}_{4}(x+t \bar{H})=0$ on account of $(3) \Rightarrow$ (2) of Proposition 2.2. Since $\lambda_{3} \neq 0$ and the leading term with 
respect to $x_{5}$ of $q$ is divisible by $r$, we have $\operatorname{deg}_{x_{3}, x_{4}, x_{5}} \bar{q}=\operatorname{deg}_{x_{4}, x_{5}} \bar{q}+1=3$. The coefficient of $t^{3}$ in $\bar{q}(x+t \bar{H})$ is of the form $x_{1} \bar{H}_{3} s\left(\bar{H}_{4}, \bar{H}_{5}\right)$, where $s$ is a quadratic form, which decomposes into linear factors. Since $\bar{H}_{3} \neq 0$, we deduce that $s\left(\bar{H}_{4}, \bar{H}_{5}\right)=0$ and that $\bar{H}_{5}$ is linearly dependent over $\mathbb{C}$ of $\bar{H}_{4}$.

(iii) By way of a linear conjugation of $H$ and the same linear conjugation of $\bar{H}$, we can obtain $\bar{H}_{5}=0$. If $\bar{H}_{3}=0$, then one can compute that $\operatorname{deg}_{x_{5}} \mathcal{J} p \cdot H$ $=\operatorname{deg}_{x_{5}} \frac{\partial}{\partial x_{4}} p \cdot \bar{H}_{4}$, which gives a contradiction to (2.3) in Proposition 2.2. Hence $\bar{H}_{3} \neq 0$. From (2.4), it follows that $\mathcal{J} \bar{H}$ is nilpotent. Since $\bar{H}_{1}=\bar{H}_{2}=\bar{H}_{5}=0$, $\mathcal{J}_{x_{3}, x_{4}}\left(\bar{H}_{3}, \bar{H}_{4}\right)$ is nilpotent as well. From van den Essen (2000, Th. 7.2.25) (see also van den Essen and Hubbers 2000), we obtain that

$$
\left(\bar{H}_{3}, \bar{H}_{4}\right)=\left(b g\left(a x_{3}-b x_{4}\right)+c, a g\left(a x_{3}-b x_{4}\right)+d\right)
$$

for certain $a, b, c \in \mathbb{C}\left[x_{1}, x_{2}, x_{5}\right]$. Hence $\operatorname{deg}_{x_{4}} \bar{H}_{3}=\operatorname{deg}_{x_{4}} \bar{H}_{4}$. Since $\operatorname{deg}_{x_{4}} p \geq$ 1 and $\operatorname{deg}_{x_{4}, x_{5}} p=1$, this is only possible if $x_{5} \mid a$ and $\operatorname{deg}_{x_{4}} p=\operatorname{deg}_{x_{4}} \bar{q}=1$. Since $\bar{q}$ be the leading and quadratic part of $q$ with respect to $\left(x_{4}, x_{5}\right)$, we deduce from $\operatorname{deg}_{x_{4}} \bar{q}=1$ that $q$ has a term which is divisible by $x_{4} x_{5}$, but no term which is divisible by $x_{4}^{2}$. So $\operatorname{deg}_{x_{4}} p=\operatorname{deg}_{x_{4}} q=1$ and the right hand side of

$$
\operatorname{deg}_{x_{5}}\left(\frac{\partial}{\partial x_{4}} p\right)=0 \text { and } \operatorname{deg}_{x_{5}}\left(\frac{\partial}{\partial x_{4}} q\right)=1
$$

follows. The left hand side of (5.3) follows from $\operatorname{deg}_{x_{4}, x_{5}} p=1$.

(iv) Since $q$ is an invariant of $x+H$, we obtain from Proposition 2.2 that $q(x+t H) \cdot H(x+t H)=q \cdot H$, and substituting $t=t q$ gives by way of (2) $\Rightarrow$ (1) of Proposition 2.2 that $x+q H$ is a quasi-translation. Since the leading coefficient with respect to $x_{4}$ of $q$ and hence also $q H_{5}$ is contained in $\mathbb{C}\left[x_{1}, x_{2}, x_{3}, x_{5}\right] \backslash \mathbb{C}\left[x_{1}, x_{2}, x_{3}\right]$, we deduce that $\operatorname{deg}_{x_{4}} q H_{5}=\operatorname{deg}_{x_{4}} \frac{\partial}{\partial x_{5}}\left(q H_{5}\right)$. On account of (2.4) in Proposition 2.2, we have $\operatorname{tr} \mathcal{J} q H=0$, so

$$
\begin{aligned}
\operatorname{deg}_{x_{4}} H_{5} & =\operatorname{deg}_{x_{4}}\left(q H_{5}\right)-\operatorname{deg}_{x_{4}} q \\
& =\operatorname{deg}_{x_{4}} \frac{\partial}{\partial x_{5}}\left(q H_{5}\right)-\operatorname{deg}_{x_{4}} q \\
& \leq \operatorname{deg}_{x_{4}}\left(q H_{1}, q H_{2}, q H_{3}, q H_{4}\right)-\operatorname{deg}_{x_{4}} q \\
& =\operatorname{deg}_{x_{4}}\left(H_{1}, H_{2}, H_{3}, H_{4}\right) \\
& =\operatorname{deg} h=3
\end{aligned}
$$

Take $k$ minimal such that the leading coefficient with respect to $x_{4}$ of $p$ is contained in $\mathbb{C}\left[x_{1}, x_{2}, \ldots, x_{k}\right]$. Since $\operatorname{deg}_{x_{4}} H_{5} \leq \operatorname{deg} h$ and $\operatorname{deg}_{x_{4}, x_{5}} p=1$, we have $\left(\frac{\partial}{\partial x_{4}}\right)^{\operatorname{deg} h+1}\left(H_{5} \frac{\partial}{\partial x_{5}} p\right)=0$. From (2.3) it follows that $\mathcal{J} p \cdot H=0$, so that we can deduce from $\operatorname{deg}_{x_{4}} p=\operatorname{deg}_{x_{4}} q=1$ that

$$
0-0=\left(\frac{\partial}{\partial x_{4}}\right)^{\operatorname{deg} h+1}(\mathcal{J} p \cdot H)-\left(\frac{\partial}{\partial x_{4}}\right)^{\operatorname{deg} h+1}\left(H_{5} \frac{\partial}{\partial x_{5}} p\right)
$$




$$
\begin{aligned}
& =(\operatorname{deg} h+1) ! \cdot \sum_{i=1}^{4} h\left(\frac{\partial}{\partial x_{4}} p, \frac{\partial}{\partial x_{4}} q\right) \frac{\partial}{\partial x_{4}} \frac{\partial}{\partial x_{i}} p \\
& =(\operatorname{deg} h+1) ! \cdot \sum_{i=1}^{k} h\left(\frac{\partial}{\partial x_{4}} p, \frac{\partial}{\partial x_{4}} q\right) \frac{\partial}{\partial x_{4}} \frac{\partial}{\partial x_{i}} p
\end{aligned}
$$

But the right hand side has degree $\operatorname{deg}_{y_{2}} h_{k}$ with respect to $x_{5}$ on account of (5.3). Contradiction, $\operatorname{so} \operatorname{deg} q \geq 5$.

\section{The kernel of the map $H$ of quasi-translations $x+H$}

In the beginning of the proof of Theorem 5.1, we have shown that for quasitranslations $x+H$ which belong to case (b) in Gordan and Nöther (1876), $\operatorname{dim} V(H)$ $=\mathrm{rk} \mathcal{J} H=3$. Hence the Zariski closure of the image of $H$ is an irreducible component of $V(H)$ for such quasi-translations. Corollary 6.4 in this section subsequently gives us several results about quasi-translations which belong to case (b) in Gordan and Nöther (1876), among which a result about such quasi-translations without linear invariants.

First we prove some geometric results about quasi-translations to obtain Theorem 6.3. Next, we use Theorem 6.3 to prove Corollary 6.4. At last, we use Theorem 6.3 to prove Corollary 6.5, which gives us the case where $n \geq 6$ of (iii) of Theorem 3.6.

Lemma 6.1 Assume $x+H$ is a quasi-translation in dimension n over $\mathbb{C}$. Let $X \subseteq \mathbb{C}^{n}$ be an irreducible variety such that $\left.H\right|_{X}$ is not the zero map, so that the Zariski closure $Y$ of the image of $\left.H\right|_{X}$ is nonzero.

Then for each $c \in X$, there exists a nonzero $p \in Y$ such that $g(c+t p)=g(c)$, for every invariant $g$ of $x+H$, where $t$ is a new indeterminate.

Proof Let $G$ be the set of invariants of $x+H$. We first prove this lemma for all $c$ in a nonempty open subset of $X$. The generic property of $c$ that we assume is that $H(c) \neq 0$. Since $\left.H\right|_{X}$ is not the zero map, we are considering a nonempty open subset of $X$ indeed. From (2.3) in Proposition 2.2, it follows that $g(x+t H)=g(x)$ for every invariant $g$ of $x+H$. Hence $g(c+t p)=g(c)$ for every $g \in G$, if we take $p=H(c) \neq 0$.

In the general case, consider the sets

$$
Z:=\left\{(c, p, b) \in X \times\left(\mathbb{C}^{n}\right)^{2} \mid g(c+t p)=g(c) \text { for every } g \in G \text { and } b^{\mathrm{t}} p=1\right\}
$$

and

$$
\tilde{Z}:=\{(c, p, b) \in Z \mid b \text { is the complex conjugate of } p\}
$$

By applying proper substitutions in $t$, we see that the image $\tilde{X}$ of the projection of $\tilde{Z}$ onto its first $n$ coordinates is equal to that of $Z$. Since $\tilde{X}$ contains an open subset of $X$, it follows from Lemma 3.1 that $\tilde{X}=X$, which gives the desired result. 
Lemma 6.2 Assume $x+H$ is a quasi-translation in dimension $n$ over $\mathbb{C}$. Let $W$ be the Zariski closure of the image of $H$. Then for any linear subspace $L$ of $\mathbb{C}^{n}$, the assertions

(1) $\operatorname{dim} L>\operatorname{dim} V(H)$;

(2) every irreducible component of $H^{-1}(L)$ has dimension greater than $\operatorname{dim} V(H)$;

(3) for each $c \in V(H)$, there exists a nonzero $p \in L \cap W$ such that $H(c+t p)=0$; satisfy $(1) \Rightarrow(2) \Rightarrow(3)$.

Proof Assume that $L$ is a linear subspace of $\mathbb{C}^{n}$.

(1) $\Rightarrow(2)$ Notice that $H^{-1}(L)$ is the zero set of $n$-dim $L$ linear forms in $H_{1}, H_{2}, \ldots$, $H_{n}$. By applying (Hartshorne 1977, Ch. I, Prop. 7.1) $n-\operatorname{dim} L-1$ times, it follows that every irreducible component of $H^{-1}(L)$ has dimension at least $\operatorname{dim} L$, which exceeds $\operatorname{dim} V(H)$ if (1) is satisfied.

(2) $\Rightarrow$ (3) Assume $H(c)=0$. Since $V(H)=H^{-1}(0) \subseteq H^{-1}(L)$, there exists an irreducible component $X$ of $H^{-1}(L)$ which contains $c$. Assuming (2), we obtain $\operatorname{dim} X>\operatorname{dim} V(H)$, whence $\left.H\right|_{X}$ is not the zero map. Hence (2) $\Rightarrow$ (3) follows from Lemma 6.1 and (1) $\Rightarrow$ (2) of Proposition 2.2.

Theorem 6.3 Assume $x+H$ is a homogeneous quasi-translation over $\mathbb{C}$. Let $W$ be the Zariski closure of the image of $H$.

Then for every irreducible component $X$ of $V(H)$ such that $\operatorname{dim}(X \cap W) \leq n-$ $\operatorname{dim} V(H), X \cap W$ has an irreducible component $Z$ of dimension $n-\operatorname{dim} V(H)$, such that $c+p \in X$ for all $c \in X$ and all $p$ in the linear span of $Z$.

Proof Let $X$ be an irreducible component of $V(H)$ such that $\operatorname{dim}(X \cap W) \leq n-$ $\operatorname{dim} V(H)$. We can take a linear subspace $M$ of $\mathbb{C}^{n}$, such that $c+p \subseteq X$ for all $c \in X$ and all $p \in M$, because $M=\{0\}^{n}$ suffices. Take $M$ as above such that $\operatorname{dim} M$ is as large as possible. Suppose first that $\operatorname{dim}(M \cap X \cap W)=n-\operatorname{dim} V(H)$. Then $\operatorname{dim}(X \cap W)=n-\operatorname{dim} V(H)$ as well, so that $X \cap W$ has an irreducible component $Z \subseteq M$ of maximum dimension $n-\operatorname{dim} V(H)$. Since $M$ contains the linear span of $Z$, it follows from the definition of $M$ that $Z$ suffices.

Suppose next that $\operatorname{dim}(M \cap X \cap W)<n-\operatorname{dim} V(H)$. Take for $L$ a generic linear subspace of $\mathbb{C}^{n}$ of dimension $\operatorname{dim} V(H)+1$, to obtain that $\operatorname{dim}(L \cap(M \cap X \cap W))=0$ and $\operatorname{dim}(L \cap(X \cap W)) \leq 1$. Since $X$ is an irreducible component of $V(H)$, the interior $X^{\circ}$ of $X$ as a closed subset of $V(H)$ is nonempty. Now take an arbitrary $c \in X^{\circ}$. On account of $(1) \Rightarrow(3)$ of Lemma 6.2, there exists a nonzero $p \in L \cap W$, such that $H(c+t p)=0$. Since $H$ is homogeneous, the set $L \cap W$ is a union of lines through the origin. Hence there exists a line $P \subseteq L \cap W$ though the origin, such that $c+P \subseteq V(H)$.

Since $c \in X^{\circ}$ and $X$ is an irreducible component of $V(H)$, we deduce that $c+P \subseteq$ $X$. In particular, $P \subseteq X$, so $P \subseteq L \cap X \cap W$. But $\operatorname{dim}(L \cap X \cap W) \leq 1$, so $L \cap X \cap W$ can only contain finitely many lines through the origin, say that $Q$ is the finite set of these lines. Since $X^{\circ}$ is dense in $X$ and $c$ was arbitrary, we can deduce that

$$
X=\bigcup_{P \in Q}\{c \in X \mid c+P \subseteq X\}
$$

Since $X$ is irreducible, there exists a $P \in Q$ such that $c+P \subseteq X$ for all $c \in X$. Therefore we can replace $M$ by $M \oplus P$, which contradicts the maximality of $\operatorname{dim} M$. 
Corollary 6.4 Assume $x+H$ is a homogeneous quasi-translation over $\mathbb{C}$, such that $\operatorname{dim} V(H) \leq 3$ and $\operatorname{gcd}\left\{H_{1}, H_{2}, \ldots, H_{n}\right\}=1$.

Then the Zariski closure $W$ of the image of $H$ is contained in $V(H)$. Furthermore, every irreducible component $X$ of $V(H)$ which is not equal to $W$ is a 3-dimensional linear subspace of $\mathbb{C}^{n}$ for which $\operatorname{dim}(X \cap W)=2$.

If $W$ has a nonzero (projective) apex $p$ and $V(H)$ has a component $X$ which does not contain $p$, then $W$ is contained in the 4-dimensional linear subspace of $\mathbb{C}^{n}$ which is spanned by $X$ and $p$.

Proof Using (2) $\Rightarrow$ (3) of Proposition 2.6 and Lemma 3.2, we deduce that $W$ is irreducible and that $W \subseteq V(H)$. Let $X$ be an irreducible component of $V(H)$ which is not equal to $W$. Since $X \neq W$ and $\operatorname{dim} V(H) \leq 3$, we have $\operatorname{dim}(X \cap W) \leq 2$. From $\operatorname{gcd}\left\{H_{1}, H_{2}, \ldots, H_{n}\right\}=1$, we deduce that $\operatorname{dim}(X \cap W) \leq 2 \leq n-\operatorname{dim} V(H)$. On account of Theorem 6.3, $X \cap W$ has an irreducible component $Z$ of dimension $n-\operatorname{dim} V(H)=2=\operatorname{dim}(X \cap W)$, such that $c+q \in X$ for all $c \in X$ and all $q$ in the linear span of $Z$.

Notice that $\operatorname{dim} X \leq \operatorname{dim} V(H) \leq 3$. Suppose that $\operatorname{dim} X \leq 2$. Then $X \subseteq W$ because $X$ is irreducible and $\operatorname{dim}(X \cap W)=2$. Since $W$ is irreducible, this contradicts the fact that $X$ is an irreducible component of $V(H)$ which is not equal to $W$. Thus $\operatorname{dim} X=3$. Let $r$ be the dimension of the linear span of $Z$. If $r \geq 3$, then $X$ contains the linear span of $r$ independent $q \in Z$, whence $X$ is equal to the linear span of $r=3$ independent $q \in Z$. If $r \leq 2$, then $r=2$ because $\operatorname{dim} Z=2$, and $X$ is the linear span of two independent $q \in Z$, and any $c \in X \backslash Z$.

Suppose that $W$ has a nonzero (projective) apex $p$ and $V(H)$ has a component $X$ which does not contain $p$. Since $\operatorname{dim}(X \cap W)=2$, there are infinitely many GN-planes spanned by $p$ and a nonzero $q \in X \cap W$. Any proper algebraic subset of $W$ can only have finitely many GN-planes, because $W$ is irreducible and $\operatorname{dim} W=3$. Hence the set of infinitely many GN-planes spanned by $p$ and a nonzero $q \in X \cap W$ is dense in $W$. It follows that $W$ is contained in the linear span of $X$ and $p$.

Corollary 6.5 Assume $x+H$ is a homogeneous quasi-translation over $\mathbb{C}$, such that $\operatorname{rk} \mathcal{J} H+\operatorname{dim} V(H) \leq n$. Then $H(c+p)=0$ for all $c \in V(H)$ and all $p$ in the linear span of the image of $H$. In particular, $x+H$ has at least $\mathrm{rk} \mathcal{J} H$ linear invariants.

Proof The case where $\operatorname{deg} H \leq 0$ is easy, so assume that $\operatorname{deg} H \geq 1$. Let $W$ be the Zariski closure of the image of $H$ and $X$ be an irreducible component of $V(H)$. From Lemma 3.2, it follows that $W$ is irreducible and that $\operatorname{dim}(X \cap W) \leq \operatorname{dim} W$ $=\operatorname{rk} \mathcal{J} H \leq n-\operatorname{dim} V(H)$. Using Theorem 6.3, we subsequently deduce that $X \cap W$ has an irreducible component $Z$ of dimension $n-\operatorname{dim} V(H)$, such that $c+p \in X$ for all $c \in X$ and all $p$ in the linear span of $Z$.

If $W \nsubseteq X$, then by the irreducibility of $W, \operatorname{dim} Z \leq \operatorname{dim}(X \cap W)<\operatorname{dim} W=$ rk $\mathcal{J} H \leq n-\operatorname{dim} V(H)$, which contradicts $\operatorname{dim} Z=n-\operatorname{dim} V(H)$. Hence $W \subseteq X$, and by irreducibility of $W$ once again, the only irreducible component of $X \cap W$ is $W$. Thus $Z=W$. Furthermore, $X$ is an arbitrary irreducible component of $V(H)$, so $c+p \in V(H)$ for all $c \in V(H)$ and all $p$ in the linear span of $W$.

Consequently, $H(c+p)=0$ for all $c \in V(H)$ and all $p$ in the linear span of the image of $H$. Furthermore, the dimension of the linear span of the image of $H$ 
does not exceed the dimension of $V(H)$. So there are at least $r:=n-\operatorname{dim} V(H)$ $\geq \operatorname{rk} \mathcal{J} H$ independent linear forms $l_{1}, l_{2}, \ldots, l_{r}$ which vanish on the image of $H$. Hence $l_{i}(H)=0$ and $l_{i}(x+H)=l_{i}(x)$ for each $i$, as desired.

Open Access This article is distributed under the terms of the Creative Commons Attribution 4.0 International License (http://creativecommons.org/licenses/by/4.0/), which permits unrestricted use, distribution, and reproduction in any medium, provided you give appropriate credit to the original author(s) and the source, provide a link to the Creative Commons license, and indicate if changes were made.

\section{References}

Ciliberto, C., Russo, F., Simis, A.: Homaloidal hypersurfaces and hypersurfaces with vanishing Hessian. Adv. Math. 218, 1759-1805 (2008)

de Bondt, M.C.: Quasi-translations and counterexamples to the homogeneous dependence problem. Proc. Am. Math. Soc. 134(10), 2849-2856 (2006) (electronic)

de Bondt, M.C.: Homogeneous Keller maps, Ph.D. thesis. Radboud University Nijmegen (2009). http:// webdoc.ubn.ru.nl/mono/b/bondt_m_de/homokema.pdf

de Bondt, M.: The strong nilpotency index of a matrix. Linear Multilinear Algebra 62(4), 486-497 (2014)

de Bondt, M.C., van de Essen, A.R.P.: Singular Hessians. J. Algebra 282(1), 195-204 (2004)

de Bondt, M.C., van den Essen, A.R.P.: The Jacobian conjecture: linear triangularization for homogeneous polynomial maps in dimension three. J. Algebra 294(1), 294-306 (2005)

Franchetta, A.: Sulle forme algebriche di $S_{4}$ aventi l'hessiana indeterminata. Rend. Mat. Appl. 5(14), 252257 (1954)

Garbagnati, A., Repetto, F.: A geometrical approach to Gordan-Nöther's and Franchetta's contributions to a question posed by Hesse. Collect. Math. 60(1), 27-41 (2009)

Gordan, P., Nöther, M.: Über die algebraische Formen, deren Hesse'sche Determinante identisch verschwindet. Math. Ann. 10, 547-568 (1876)

Hartshorne, R.: Algebraic Geometry. Graduate Texts in Mathematics, vol. 52. Springer, New York (1977)

Liu, D.: On homogeneous quasi-translations in dimension five. Int. Math. Forum 6(53-56), 2655-2664 (2011)

Lossen, C.: When does the Hessian determinant vanish identically? (On Gordan and Nöther's proof of Hesse's claim). Bull. Braz. Math. Soc. (N.S.) 35(1), 71-82 (2004)

Mumford, D.: The Red Book of Varieties and Schemes, second expanded edition. Includes the Michigan Lectures (1974) on Curves and Their Jacobians. With Contributions by Enrico Arbarello. Lecture Notes in Mathematics, vol. 1358. Springer, Berlin (1999)

O’Meara, K.C., Clark, J., Vinsonhaler, C.I.: Advanced Topics in Linear Algebra. Weaving Matrix Problems Through the Weyr Form. Oxford University Press, Oxford (2011)

Russo, F.: On the Geometry of Some Special Projective Varieties. Lecture Notes of the Unione Matematica Italiana. Springer, Berlin (2016)

van den Essen, A.: Polynomial Automorphisms and the Jacobian Conjecture. Progress in Mathematics, vol. 190. Birkhäuser, Berlin (2000)

van den Essen, A.R.P., Hubbers, E.-M.G.M.: A new class of invertible polynomial maps. J. Algebra 187(1), 214-226 (1997)

Watanabe, J.: On the theory of Gordan-Noether on homogeneous forms with zero Hessian. Proc. Sch. Sci. Tokai Univ. 49, 1-21 (2014) 